\title{
VEHICLE AND FLEET RANDOM EFFECTS IN A MODEL OF INSURANCE RATING FOR FLEETS OF VEHICLES
}

\author{
BY \\ Jean-François Angers ${ }^{1}$, Denise Desjardins'2, \\ GEORGES DIONNE ${ }^{3}$ AND FranÇOIS GUERTIN ${ }^{4}$
}

\begin{abstract}
We are proposing a parametric model to rate insurance for vehicles belonging to a fleet. The tables of premiums presented take into account past vehicle accidents, observable characteristics of the vehicles and fleets, and violations of the road-safety code committed by drivers and carriers. The premiums are also adjusted according to accidents accumulated by the fleets over time. The proposed model accounts directly for explicit changes in the various components of the probability of accidents. It represents an extension of bonus malus-type automobile insurance models for individual premiums (Lemaire, 1985; Dionne and Vanasse, 1989 and 1992; Pinquet, 1997 and 1998; Frangos and Vrontos, 2001; Purcaru and Denuit, 2003). The extension adds a fleet effect to the vehicle effect so as to account for the impact that the unobservable characteristics or actions of carriers can have on truck accident rates. This form of rating makes it possible to visualize what impact the behaviors of owners and drivers can have on the predicted rate of accidents and, consequently, on premiums. The results are compared to those of the semiparametric approach.
\end{abstract}

\section{KEYWORDS}

Fleet of vehicles, random effects, vehicle effect, fleet effect, insurance rating, behaviors of owners and drivers, Poisson, gamma, Dirichlet, parametric model.

\section{INTRODUCTION}

Very few studies have analyzed systematically the risks of accidents for vehicle fleets. Marie-Jeanne (1994) developed a rating model based on the size of the

\footnotetext{
Département de mathématiques et de statistique, Université de Montréal and CRT.

2 CRT, Université de Montréal.

3 HEC Montréal, CRT, CIRPÉE, and THEMA (France).

${ }^{4}$ RQCHP and CRT, Université de Montréal.
} 
fleet and Teugels and Sundt (1991) proposed rating based on the aggregated loss of the fleet. Other researchers have confined themselves to studying the drivers of vehicles to obtain a portrait of the risks posed by a carrier (Dionne et al., 2001). This amounts to forgetting that firms' owners or management can also affect the accident rates of their vehicles. Decisions regarding driving hours, spending on vehicle maintenance, and guidelines for loading or securing cargo in vehicles can have repercussions on road safety. Dionne, Desjardins, and Pinquet (1999 and 2001) developed bonus malus models that use a semiparametric approach to take into account the behaviors of both the drivers and owners of vehicles. In this article, we propose a parametric model.

Measuring the risks associated with fleets of vehicles is difficult for a number of reasons. For one, the units composing the fleets must be defined. Should we do this by observing drivers or vehicles? We answered that question by opting for vehicles: with information readily available from insurers, the link between vehicles and carriers can be made continuously. Linking information on drivers to carriers is, in contrast, very costly, since the movements of drivers from one fleet to another are not systematically recorded, whereas licensing and insurance contracting keep track of vehicles as they move among fleets. The vehicles are taken to represent different individual risks. These risks are influenced by the observable and unobservable characteristics of the vehicles, the drivers using them, and the carriers who own or lease them. It is thus essential to use care in modeling these different sources of information.

Another difficulty is weighting the information obtained on individuals and fleets for insurance rating purposes. An adequate model for rating the risks of fleets must integrate the behaviors of drivers with those of owners so as to introduce incentives for safety tailored to the various levels of decisions to be made when facing hierarchical moral hazard (Moses and Savage, 1994, 1996; Fluet, 1999; Winter, 2000).

We are proposing a new rating model for vehicles belonging to a fleet. The model is a parametric one which can account directly for both observable and unobservable characteristics of the vehicles, drivers, and owners associated with a particular vehicle fleet. The model proposed is a direct extension of bonus malus-type automobile insurance models (Lemaire 1985, 1995; Dionne and Vanasse 1989 and 1992; Pinquet 1997, 1998; Frangos and Vrontos 2001; Purcaru and Denuit 2003) to individual premiums (see Pinquet, 2000, for a review of the literature). The extension adds a random fleet effect to the vehicle effect in the model, in order to take into account the unobservable effects of carriers, vehicles, and their drivers on truck accident rates in the Bayesian or a posteriori calculation of premiums. Observable variables characterizing vehicles, fleets, and the road-safety behavior of both drivers and carriers are used in evaluating the a priori risks of different vehicles.

In the following section, we develop statistical models to estimate accident probabilities for vehicles belonging to fleets of various sizes. Estimation results are also presented. Section 3 proposes the optimal bonus malus system integrating both fleet and vehicle effects. Section 4 presents different premium 
tables and compares the results to those of the semiparametric approach. Section 5 discusses possible extensions of the model.

\section{Statistical MODELS}

Our methodology is divided into two steps. In the first step, we use an econometric model to evaluate the accident probabilities for the vehicles of carriers. As a priori information, we shall use estimated parameters to calculate insurance premiums. These parameters take into account the information available on the observable characteristics of vehicles and fleets as well as that on traffic violations by drivers and carriers. In order to take unobservable characteristics and actions into account for purposes of rating, we shall use the residuals of the econometric estimations. One of the article's contributions consists in proposing a new model for estimating accident probabilities, a model capable of explicitly isolating the fleet effect from the vehicle effect. In a second step, we propose a bonus malus system which can use both the a priori information obtained from the estimated parameters and the a posteriori information obtained from residuals of the estimations of vehicle accident distributions. In order to show what contribution the different effects make to insurance premiums, we shall distinguish between one-vehicle and two-vehicle carriers and then generalize the model to carriers with more than two vehicles.

\subsection{Econometric model for estimating distributions of vehicle accidents}

Most econometric models applied to discrete (or countable) variables are based on the Poisson distribution, where probability $P\left(y_{f i}^{j} \mid \lambda_{f i}^{j}\right)$ that a vehicle $i$ belonging to fleet $f$ will be involved in accidents at period $j$ can be represented by the following expression:

$$
P\left(y_{f i}^{j} \mid \lambda_{f i}^{j}\right)=\frac{e^{-\lambda_{f i}^{j}}\left(\lambda_{f i}^{j}\right)^{y_{f i}^{j}}}{\Gamma\left(y_{f i}^{j}+1\right)} .
$$

With the Poisson law, we obtain that the mathematical expectation of the number of accidents is equal to the variance $E\left(Y_{f i}^{j}\right)=\operatorname{Var}\left(Y_{f i}^{j}\right)=\lambda_{f i}^{j}$ where $Y_{f i}^{j}$ is the number of accidents for truck $i$ belonging to fleet $f$ at period $j$ and $\lambda_{f}^{j}$ is the parameter of the Poisson distribution. This modeling implicitly supposes that the distribution of accidents can be entirely explained by observable heterogeneity, which cancels any need for a bonus malus system.

Let us now suppose that an unobservable heterogeneity exists owing to certain characteristics or actions not observable by the insurer. Suppose that $\lambda_{f i}^{j}=$ $\gamma_{f i}^{j} \alpha_{f} \theta_{f i}$ with $\gamma_{f i}^{j}=d_{f i}^{j} e^{X_{f i}^{j} \beta}$ where $d_{f i}^{j}$ measures the number of days that vehicle $i$ of fleet $f$ is authorized to circulate during period $j$, divided by the number of 
total days in period $j$. This measures the exposure to the risk of accident in period $j$. Using the exponential function to define $\gamma_{f i}^{j}$ allows us to ensure the non-negativity of $\lambda_{f i}^{j}$. The vector $X_{f i}^{j}=\left(x_{f i 1}^{j}, \ldots, x_{f i p}^{j}\right)$ contains the $p$ characteristics of truck $i$ in fleet $f$ observed at period $j$; this vector contains specific information on the vehicle and on other characteristics of the fleet. $\beta$ is a vector of parameters to be estimated. As usual, the parameters are neither functions of fleet $f$ nor of period $j$, but the $X_{f i}^{j}$ are functions of $f$ and $j$, so the $\gamma_{f i}^{j}$ are adjusted when the values of $f$ and $j$ change. Parameter $\alpha_{f}$ is the random effect associated with fleet $f$, that is, the unobservable risk attributable to the fleet; whereas parameter $\theta_{f i}$ is the random effect of truck $i$ in fleet $f . \alpha_{f}$ is the usual random firm-specific effect in econometric analysis. It captures unobservable factors of the fleet orthogonal to other observed fleet variables. We suppose that $\sum_{i=1}^{I_{f}} \theta_{f i}=1$ where $I_{f}$ is the total number of vehicles in fleet $f$. In other terms, $\theta_{f i}$ is the proportion of the risk for fleet $f$ which can be attributed to vehicle $i$; the total unobservable risk for vehicle $i$ of fleet $f$ is defined by $\alpha_{f} \theta_{f i}$. It should be noted that when fleet $f$ has only one vehicle such that $I_{f}=1, \theta_{f 1}=1$, by definition. This means that the risk attributable to vehicle corresponds to that of the fleet, from which it follows that $\lambda_{f 1}^{j}=\gamma_{f 1}^{j} \alpha_{f}$.

We make the hypothesis that $\theta_{f}$ follows a Dirichlet parametric distribution with parameters $\left(v_{1}, v_{2}, \ldots, v_{I_{f}}\right)$ and that $\alpha_{f}$ follows a gamma distribution with parameters $\left(I_{f} \tau_{f}, \tau_{f}\right)$. This parametrization makes it possible to obtain a mean fleet effect that increases with the number of vehicles in the fleet.

\subsubsection{Size-1 carrier}

In this case, $\theta_{f i}=1$ and $\alpha_{f}$ follows a gamma distribution with parameters $\left(\tau_{f}, \tau_{f}\right)$. For period $j$, the distribution of the number of accidents for a fleet with one vehicle is given by:

$$
P\left(y_{f 1}^{j} \mid \gamma_{f 1}^{j}\right)=\int_{0}^{1} P\left(y_{f 1}^{j} \mid \alpha_{f}, \gamma_{f 1}^{j}\right) f\left(\alpha_{f}\right) d \alpha_{f},
$$

which, assuming that $\alpha_{f}$ follows a gamma distribution with density $\frac{\left(\tau_{f}\right)^{\tau_{f}}\left(\alpha_{f}\right)_{f}^{\tau_{f}-1} e^{-\alpha_{f} \tau_{f}}}{\Gamma\left(\tau_{f}\right)}$, can be rewritten as follows:

$$
P\left(y_{f 1}^{j} \mid \gamma_{f 1}^{j}\right)=\frac{\Gamma\left(\tau_{f}+y_{f 1}^{j}\right)}{\Gamma\left(y_{f 1}^{j}+1\right) \Gamma\left(\tau_{f}\right)}\left(\frac{\tau_{f}}{\tau_{f}+\gamma_{f 1}^{j}}\right)^{\tau_{f}}\left(\frac{\gamma_{f 1}^{j}}{\tau_{f}+\gamma_{f 1}^{j}}\right)^{y_{f 1}^{j}} .
$$

This distribution is the standard negative binomial distribution that has been used fairly often in the literature (Lemaire, 1985; Dionne and Vanasse, 1989; Hausman et al., 1984; Gouriéroux et al., 1984; Gouriéroux, 1999). It is capable of modeling unobservable heterogeneity and of introducing a bonus malus 
system for individual observations. On the other hand, it is not directly applicable when estimating the probability of accidents for vehicles belonging to a fleet, as it cannot isolate the fleet effect from the vehicle effect. We now present our generalization of this basic model, starting with the simple case of a fleet composed of two vehicles.

\subsubsection{Carrier with 2 vehicles}

The joint probability of the number of accidents at period $j$ for the two vehicles in fleet $f$ is given by:

$$
P\left(y_{f 1}^{j}, y_{f 2}^{j} \mid \gamma_{f 1}^{j}, \gamma_{f 2}^{j}\right)=\int_{0}^{1} P\left(y_{f 1}^{j}, y_{f 2}^{j} \mid \theta_{f}, \gamma_{f 1}^{j}, \gamma_{f 2}^{j}\right) d \theta_{f}
$$

where

$$
\theta_{f}=\theta_{f 1} \text { and } 1-\theta_{f}=\theta_{f 2} .
$$

Conditionally on $\theta_{f}$, the joint probability of accident is equal to:

$$
\begin{aligned}
& P\left(y_{f 1}^{j}, y_{f 2}^{j} \mid \theta_{f}, \gamma_{f 1}^{j}, \gamma_{f 2}^{j}\right)= \\
& {\left[\frac{\left(\gamma_{f 1}^{j}\right)^{y_{1}^{j}}\left(\gamma_{f 2}^{j}\right)^{y_{j 2}^{j}}\left(\theta_{f}\right)^{y_{f 1}^{j}}\left(1-\theta_{f}\right)^{y_{f 2}^{j}} \tau_{f}^{2 \tau_{f}}}{\Gamma\left(y_{f 1}^{j}+1\right) \Gamma\left(y_{f 2}^{j}+1\right) \Gamma\left(2 \tau_{f}\right)}\right] \int_{0}^{\infty}\left(\alpha_{f}\right)^{2 \tau_{f}+\sum_{i=1}^{2} y_{f}^{j}-1} e^{-\alpha_{f}\left(\tau_{f}+\theta_{f} \gamma_{\gamma_{1}}^{j}+\left(1-\theta_{f}\right) \gamma_{12}^{j}\right)} d \alpha_{f}}
\end{aligned}
$$

where $\alpha_{f}$ follows a gamma distribution with parameters $\left(2 \tau_{f}, \tau_{f}\right)$. So its density function is equal to:

$$
\frac{\left(\tau_{f}\right)^{2 \tau_{f}}\left(\alpha_{f}\right)^{2 \tau_{f}-1} e^{-\alpha_{f} \tau_{f}}}{\Gamma\left(2 \tau_{f}\right)} .
$$

By integrating (3) with respect to $\alpha_{f}$ and substituting the value of $P\left(y_{f 1}^{j}, y_{f 2}^{j} \mid \theta_{f}, \gamma_{f 1}^{j}, \gamma_{f 2}^{j}\right)$ in (2), we obtain:

$$
\begin{aligned}
& P\left(y_{f 1}^{j}, y_{f 2}^{j} \mid \gamma_{f 1}^{j}, \gamma_{f 2}^{j}\right)= \\
& \int_{0}^{1} \frac{\left(\gamma_{f 1}^{j}\right)^{y_{f 1}^{j}}\left(\gamma_{f 2}^{j}\right)^{y_{f 2}^{j}}\left(\theta_{f}\right)^{y_{f 1}^{j}}\left(1-\theta_{f}\right)^{y_{j 2}^{j}} \tau_{f}^{2 \tau_{f}}}{\Gamma\left(y_{f 1}^{j}+1\right) \Gamma\left(y_{f 2}^{j}+1\right) \Gamma\left(2 \tau_{f}\right)} \frac{\Gamma\left(2 \tau_{f}+\sum_{i=1}^{2} y_{f i}^{j}\right)}{\left(\tau_{f}+\theta_{f} \gamma_{f 1}^{j}+\left(1-\theta_{f}\right) \gamma_{f 2}^{j}\right)^{2 \tau_{f}+\sum_{i=1}^{2} y_{f i}^{j}} f\left(\theta_{f}\right) d \theta_{f} .} .
\end{aligned}
$$


In order to estimate the probabilities of accident with a parametric approach, we must now make the distribution of $\theta_{f}$ more explicit. Since fleets have two vehicles, we suppose that the vehicle effect follows a Dirichlet distribution, with two parameters. Note that the Dirichlet distribution with two parameters is the beta distribution. By replacing the density function $f\left(\theta_{f}\right)$ in equation (4) with the density of a Dirichlet with parameters $\left(v_{1}, v_{2}\right)$ or a standard beta distribution

$$
f\left(\theta_{f}\right)=\frac{\Gamma\left(\sum_{i=1}^{2} v_{i}\right)}{\prod_{i=1}^{2} \Gamma\left(v_{i}\right)}\left(\theta_{f}\right)^{v_{1}-1}\left(1-\theta_{f}\right)^{v_{2}-1},
$$

we obtain:

$$
\begin{aligned}
P\left(y_{f 1}^{j}, y_{f 2}^{j} \mid \gamma_{f 1}^{j}, \gamma_{f 2}^{j}\right) & =\frac{\left(\gamma_{f 1}^{j}\right)^{v_{f 1}^{j}}\left(\gamma_{f 2}^{j}\right)^{y_{j 2}^{j}} \tau_{f}^{2 \tau_{f}} \Gamma\left(2 \tau_{f}+\sum_{i=1}^{2} y_{f i}^{j}\right)}{\Gamma\left(y_{f 1}^{j}+1\right) \Gamma\left(y_{f 2}^{j}+1\right) \Gamma\left(2 \tau_{f}\right)}\left[\frac{\Gamma\left(v_{1}+v_{2}\right)}{\Gamma\left(v_{1}\right) \Gamma\left(v_{2}\right)}\right] \\
& \times \int_{0}^{1} \frac{\left(\theta_{f}\right)^{v_{1}+y_{f 1}^{j}-1}\left(1-\theta_{f}\right)^{v_{2}+y_{f 2}^{j}-1}}{\left(\tau_{f}+\theta_{f} \gamma_{f 1}^{j}+\left(1-\theta_{f}\right) \gamma_{f 2}^{j}\right)^{2 \tau_{f}+\sum_{i=1}^{2} y_{f i}^{j}} d \theta_{f} .}
\end{aligned}
$$

where $\times$ is the multiplicative operator. We must evaluate the integral:

$$
\int_{0}^{1} \frac{\left(\theta_{f}\right)^{v_{1}+y_{f 1}^{j}-1}\left(1-\theta_{f}\right)^{v_{2}+y_{f 2}^{j}-1}}{\left(\tau_{f}+\theta_{f} \gamma_{f 1}^{j}+\left(1-\theta_{f}\right) \gamma_{f 2}^{j}\right)^{2 \tau_{f}+\sum_{i=1}^{2} y_{f i}^{j}}} d \theta_{f},
$$

to compute the value of the joint probability in (5). To do so, let's write the expression $\tau_{f}+\theta_{f} \gamma_{f 1}^{j}+\left(1-\theta_{f}\right) \gamma_{f 2}^{j}$ of the denominator as follows:

$$
\left(\tau_{f}+\gamma_{f 2}^{j}\right)\left[1-\left(\frac{\gamma_{f 2}^{j}-\gamma_{f 1}^{j}}{\tau_{f}+\gamma_{f 2}^{j}}\right) \theta_{f}\right],
$$

which permits us to rewrite the integral in (5):

$$
\begin{array}{r}
\int_{0}^{1} \frac{\left(\theta_{f}\right)^{v_{1}+y_{f 1}^{j}-1}\left(1-\theta_{f}\right)^{v_{2}+y_{f 2}^{j}-1}}{\left.\left(\tau_{f}+\gamma_{f 2}^{j}\right)\left[1-\left(\frac{\gamma_{f 2}^{j}-\gamma_{f 1}^{j}}{\tau_{f}+\gamma_{f 2}^{j}}\right) \theta_{f}\right]\right)^{2 \tau f+\sum_{i=1}^{2} \gamma_{i j}^{j}}} d \theta_{f}=\frac{1}{\left(\tau_{f}+\gamma_{f 2}^{j}\right)^{2 \tau_{f}+\sum_{i=1}^{2} y_{f}^{j}}}\left[\frac{\Gamma\left(v_{1}+y_{f 1}^{j}\right) \Gamma\left(v_{2}+y_{f 2}^{j}\right)}{\Gamma\left(v_{1}+v_{2}+y_{f 1}^{j}+y_{f 2}^{j}\right)}\right] \\
\times{ }_{2} F_{1}\left(v_{1}+y_{f 1}^{j} ; 2 \tau_{f}+y_{f 1}^{j}+y_{f 2}^{j} ; v_{1}+v_{2}+y_{f 1}^{j}+y_{f 2}^{j} ;\left(\frac{\gamma_{f 2}^{j}-\gamma_{f 1}^{j}}{\tau_{f}+\gamma_{f 2}^{j}}\right)\right)
\end{array}
$$


${ }_{2} F_{1}$ is a hypergeometric function whose value is equal to:

$$
1+\sum_{\ell=1}^{\infty}\left[\frac{\left(v_{1}+y_{f 1}^{j}\right)^{[\ell]}\left(2 \tau_{f}+\sum_{i=1}^{2} y_{f i}^{j}\right)^{[\ell]}}{\left(\sum_{i=1}^{2}\left(v_{1}+y_{f i}^{j}\right)\right)^{[\ell]}} \frac{\left(\frac{\gamma_{f 2}^{j}-\gamma_{f 1}^{j}}{\tau_{f}+\gamma_{f 2}^{j}}\right)^{\ell}}{\ell !}\right],
$$

with $h^{[\ell]}=h(h+1) \cdots(h+\ell+1)$, an increasing factorial function (Gradshteyn and Ryzhik, 1980, Section 9.1). The function is available in the GSL package.

The distribution of the number of accidents observed at period $j$ for the two vehicles in fleet $f$ is now given by:

$$
\begin{aligned}
P\left(y_{f 1}^{j}, y_{f 2}^{j} \mid \gamma_{f 1}^{j}, \gamma_{f 2}^{j}\right) & =\frac{\left(\gamma_{f 1}^{j}\right)^{y_{f 1}^{j}}\left(\gamma_{f 2}^{j}\right)^{y_{f 2}^{j}} \tau_{f}^{2 \tau_{f}} \Gamma\left(2 \tau_{f}+\sum_{i=1}^{2} y_{f i}^{j}\right) \Gamma\left(v_{1}+v_{2}\right)}{\Gamma\left(y_{f 1}^{j}+1\right) \Gamma\left(y_{f 2}^{j}+1\right) \Gamma\left(2 \tau_{f}\right) \Gamma\left(v_{1}\right) \Gamma\left(v_{2}\right)} \\
& \times \frac{\Gamma\left(v_{1}+y_{f 1}^{j}\right) \Gamma\left(v_{2}+y_{f 2}^{j}\right)}{\left(\tau_{f}+\gamma_{f 2}^{j}\right)^{2 \tau_{f}+\sum_{i=1}^{2} y_{f}^{j}} \Gamma\left(v_{1}+v_{2}+y_{f 1}^{j}+y_{f 2}^{j}\right)} \\
& \times{ }_{2} F_{1}\left(v_{1}+y_{f 1}^{j} ; 2 \tau_{f}+y_{f 1}^{j}+y_{f 2}^{j} ; v_{1}+v_{2}+y_{f 1}^{j}+y_{f 2}^{j} ;\left(\frac{\gamma_{f 2}^{j}-\gamma_{f 1}^{j}}{\tau_{f}+\gamma_{f 2}^{j}}\right)\right) .
\end{aligned}
$$

We now generalize the model to a fleet of $I_{f}$ vehicles.

\subsubsection{Carrier with more than 2 vehicles}

The joint distribution of the number of accidents at period $j$ for the $I_{f}$ vehicles in fleet $f$ is given by:

$$
\begin{aligned}
& P\left(y_{f 1}^{j}, \cdots, y_{f I_{f}}^{j} \mid \gamma_{f 1}^{j}, \cdots, \gamma_{f I_{f}}^{j}\right)= \\
& \int_{\substack{\sum_{i f} \theta_{f i}=1 \\
i=1}} \cdots \int P\left(y_{f 1}^{j}, \cdots, y_{f I_{f}}^{j} \mid \theta_{f 1}, \cdots, \theta_{f I_{f}-1}, \gamma_{f 1}^{j}, \cdots, \gamma_{f I_{f}}^{j}\right) f\left(\theta_{f 1}, \cdots, \theta_{f I_{f}}\right) d \theta_{f 1} \cdots d \theta_{f I_{f}-1}
\end{aligned}
$$

where

$$
\theta_{f I_{f}}=1-\sum_{i=1}^{I_{f}-1} \theta_{f i}
$$


We can rewrite the conditional probability in (6) as:

$$
\begin{aligned}
& P\left(y_{f 1}^{j}, \cdots, y_{f I_{f}}^{j} \mid \theta_{f 1}, \cdots, \theta_{f I_{f}-1}, \gamma_{f 1}^{j}, \cdots, \gamma_{f I_{f}}^{j}\right)= \\
& \int_{0}^{\infty} P\left(y_{f 1}^{j}, \cdots, y_{f I_{f}}^{j} \mid \alpha_{f}, \theta_{f 1}, \cdots, \theta_{f I_{f}-1}, \gamma_{f 1}^{j}, \cdots, \gamma_{f I_{f}}^{j}\right) f\left(\alpha_{f}\right) d \alpha_{f},
\end{aligned}
$$

where $\alpha_{f}$ follows a gamma distribution with parameters $\left(I_{f} \tau_{f}, \tau_{f}\right)$. By integrating with respect to $\alpha_{f}$, we obtain a Dirichlet compound multinomial distribution (Johnson and Kotz, 1969) whose joint conditional probability of accidents is equal to:

$$
\begin{aligned}
& P\left(y_{f 1}^{j}, \cdots, y_{f I_{f}}^{j} \mid \theta_{f 1}, \cdots, \theta_{f I_{f}-1}, \gamma_{f 1}^{j}, \cdots, \gamma_{f I_{f}}^{j}\right)= \\
& {\left[\prod_{i=1}^{I_{f}} \frac{\left(\gamma_{f 1}^{j}\right)^{y_{f i}^{j}}\left(\theta_{f i}\right)^{y_{f i}^{j}}}{\Gamma\left(y_{f i}^{j}+1\right)}\right] \frac{\tau_{f}^{I_{f} \tau_{f}}}{\Gamma\left(I_{f} \tau_{f}\right)} \frac{\Gamma\left(I_{f} \tau_{f}+\sum_{i=1}^{I_{f}} y_{f i}^{j}\right)}{\left.\tau_{f}+\sum_{i=1}^{I_{f}} \theta_{f i} \gamma_{f i}^{j}\right)^{I_{f} \tau_{f}+\sum_{i=1}^{I_{f i}} y_{f i}^{j}}} . }
\end{aligned}
$$

Thus, by replacing $P\left(y_{f 1}^{j}, \cdots, y_{f I_{f}}^{j} \mid \theta_{f 1}, \cdots, \theta_{f I_{f}-1}, \gamma_{f 1}^{j}, \cdots, \gamma_{f I_{f}}^{j}\right)$ in equation (6) by its value given in (7) and by replacing the density function $f\left(\theta_{f 1}, \cdots, \theta_{f I_{f}}\right)$ by the density of a parametric Dirichlet $\left(v_{1}, v_{2}, \ldots, v_{I_{f}}\right)$, we obtain the following expression:

$$
\begin{aligned}
P\left(y_{f 1}^{j}, \cdots, y_{f I_{f}}^{j} \mid \gamma_{f 1}^{j}, \cdots, \gamma_{f I_{f}}^{j}\right) & =\left[\prod_{i=1}^{I_{f}} \frac{\left(\gamma_{f i}^{j}\right)^{y_{f i}^{j}}}{\Gamma\left(y_{f i}^{j}+1\right)}\right] \frac{\tau^{I_{f} \tau_{f}} \Gamma\left(I_{f} \tau_{f}+\sum_{i=1}^{I_{f}} y_{f i}^{j}\right) \Gamma\left(\sum_{i=1}^{I_{f}} v_{1}\right)}{\Gamma\left(I_{f} \tau_{f}\right) \prod_{i=1}^{I_{f}} \Gamma\left(v_{1}\right)} \\
& \times \int_{\sum_{i=1} \theta_{f i}=1} \cdots \int \frac{\prod_{i=1}^{I_{f}}\left(\theta_{f i}\right)^{v_{i}+y_{f i}^{j}-1}}{\left(\tau_{f}+\sum_{i=1}^{I_{f}} \theta_{f i} \gamma_{f i}^{j}\right)^{I_{f} \tau_{f}+\sum_{i=1}^{I_{f}} y_{f j}^{j}} d \theta_{f 1} \cdots d \theta_{f I_{f}-1} .} .
\end{aligned}
$$

We must now estimate the multidimensional integral of equation (8) in order to estimate the model's parameters. Three possibilities are now open. They are discussed in detail in Angers et al. (2004). Here we summarize the main results.

1. The first possibility, which greatly simplifies the calculations, is to suppose that all the $\gamma_{f i}^{j}$ of the $I_{f}$ vehicles are identical.

This first scenario supposes implicitly that all the vehicles in the fleet represent identical a priori risks, which is probably a very strong hypothesis 
since, as we shall see, several variables distinguishing the vehicles and the behaviors of both the drivers and the fleet managers are significant in estimating the probabilities of accidents. Another possibility is to divide the vehicles into different risk groups, as is done by insurers when classifying risks.

2. Under the second possibility, we can separate the vehicles into two groups, for example, and define $G_{1}=1, \cdots, g$ as all the vehicles in the first group with $\sum_{i=1}^{g} \gamma_{f i}$

$\gamma_{f g 1}^{j}=\frac{\sum_{i=1}}{g}$ and $G_{2}=g+1, \cdots, I_{f}$ as all the vehicles in the second group with $\gamma_{f g 2}^{j}=\frac{\sum_{i=g+1}^{I_{f}} \gamma_{f i}^{j}}{I_{f}-g}$. The integral in (8) can thus be approximated by:

$$
\int_{\sum_{i=1}^{I_{f i}} \theta_{f_{i}}=1} \cdots \int \frac{\left[\prod_{i=1}^{g}\left(\theta_{f i}\right)^{c_{i}-1} \prod_{i=g+1}^{I_{f}}\left(\theta_{f i}\right)^{c_{i}-1}\right]}{\left(\tau_{f}+\gamma_{f g 1}^{j} \sum_{i=1}^{g} \theta_{f i}+\gamma_{f g 2}^{j} \sum_{i=g+1}^{I_{f}} \theta_{f i}\right)^{d}} d \theta_{f 1} \cdots d \theta_{f I_{f}-1}
$$

with

$$
c_{i}=v_{i}+y_{f i}^{j} \text { and } d=I_{f} \tau_{f}+\sum_{i=1}^{I_{f}} y_{f i}^{j}
$$

Taking that

$$
u_{i}=\frac{\theta_{f i}}{\sum_{i=1}^{g} \theta_{f i}} i=1, \cdots, g-1 ; v=\sum_{i=1}^{g} \theta_{f i} \text { and } w_{i}=\frac{\theta_{f i}}{1-\sum_{i=1}^{g} \theta_{f i}} i=g+1, \cdots, I_{f} \text {, }
$$

we can rewrite (9) and substitute the new expression in equation (8) to obtain an approximation for the distribution of the number of accidents at period $j$ of the vehicles in fleet $f$ :

$$
\begin{aligned}
& P\left(y_{f 1}^{j}, \cdots, y_{f I_{f}}^{j} \mid \gamma_{f 1}^{j}, \cdots, \gamma_{f I_{f}}^{j}\right) \approx \\
& \prod_{i=1}^{I_{f}}\left(\frac{\left(\gamma_{f i}^{j}\right)^{v_{f}^{j}} \Gamma\left(v_{i}+y_{f i}^{j}\right)}{\Gamma\left(y_{f i}^{j}+1\right) \Gamma\left(v_{i}\right)}\right)\left(\tau_{f}\right)^{I_{f} \tau_{f}} \frac{\Gamma\left(I_{f} \tau_{f}+\sum_{i=1}^{I_{f}} y_{f i}^{j}\right)}{\Gamma\left(I_{f} \tau_{f}\right)}\left(\frac{1}{\tau_{f}+\gamma_{f g 2}^{j}}\right)^{I_{f} \tau_{f}+\sum_{i=1}^{f_{f}} y_{f}^{j}} \frac{\Gamma\left(\sum_{i=1}^{I_{f}} v_{i}\right)}{\Gamma\left(\sum_{i=1}^{I_{f}}\left(v_{i}+y_{f i}^{j}\right)\right)} \\
& \times{ }_{2} F_{1}\left(\sum_{i=1}^{g}\left(v_{i}+y_{f i}^{j}\right), I_{f} \tau_{f}+\sum_{i=1}^{I_{f}} y_{f i}^{j}, \sum_{i=1}^{I_{f}}\left(v_{i}+y_{f i}^{j}\right), \frac{\gamma_{f g 2}^{j}-\gamma_{f g 1}^{j}}{\tau_{f}+\gamma_{f g 2}^{j}}\right),
\end{aligned}
$$


where ${ }_{2} F_{1}$ is a hypergeometric function as defined in section 2.1.2. This procedure in estimating the integral can be generalized to several homogeneous groups, but it is not obvious that more precision will be gained. In Appendix A, we present the details of the approximation error. The accuracy of the approximation can also be evaluated by comparing the estimation results with the Monte Carlo method of estimation.

3. We can also estimate the integral in (8) by the Monte Carlo method, using the function $h(\underset{\sim}{\theta})$ (Lange, 1999) with $\underset{\sim}{\theta}=\theta_{f 1}, \ldots, \theta_{f I_{f}}$, and such that:

$$
\begin{aligned}
& \int_{\sum_{i=1}^{I_{f}} \theta_{f_{i}}=1} \cdots \int g(\underset{\sim}{\theta}) d \underset{\sim}{\theta}=\int_{\sum_{i=1}^{I_{f}} \theta_{f_{i}}=1} \cdots \int \frac{g(\underset{\sim}{\theta})}{h(\underset{\sim}{\theta})} h(\underset{\sim}{\theta}) d \underset{\sim}{\theta}= \\
& \int_{\sum_{i=1}^{I_{f}} \theta_{f_{i}}=1} \cdots \int w(\underset{\sim}{\theta}) h(\underset{\sim}{\theta}) d \underset{\sim}{\theta} \approx \frac{1}{N} \sum_{l=1}^{N} w\left({\underset{\sim}{\sim}}^{l}\right)
\end{aligned}
$$

with

$$
w(\underset{\sim}{\theta})=\frac{g(\underset{\sim}{\theta})}{h(\underset{\sim}{\theta})} .
$$

Writing:

$$
h(\underset{\sim}{\theta})=\frac{\Gamma\left(v_{i}+\sum_{i=1}^{I_{f}} y_{f i}^{j}\right)}{\prod_{i=1}^{I_{f}} \Gamma\left(v_{i}+y_{f i}^{j}\right)} \prod_{i=1}^{I_{f}}\left(\theta_{f i}\right)^{v_{i}+y_{f i}^{j}-1},
$$

we can rewrite the expression of the multivariate integral by multiplying its numerator and denominator by the $h(\underset{\sim}{)})$ function defined above. After some simplifications, the expression becomes:

$$
\begin{aligned}
\int_{\sum_{i=1}^{I_{f}} \theta_{f i}=1} \cdots & \frac{1}{\left(\sum_{i=1}^{I_{f}}\left(\tau_{f}+\gamma_{f i}^{j}\right) \theta_{f i}\right)^{I_{f} \tau_{f}+\sum_{i=1}^{I_{f}} y_{f i}^{j}} \frac{\sum_{i=1}\left(v_{i}+\sum_{i=1}^{I_{f}} y_{f i}^{j}\right)}{\prod_{i=1}^{I_{f}} \Gamma\left(v_{i}+y_{f i}^{j}\right)}} \\
\times & \frac{\Gamma\left(v_{i}+\sum_{i=1}^{I_{f}} y_{f i}^{j}\right)}{\prod_{i=1}^{I_{f}} \Gamma\left(v_{i}+y_{f i}^{j}\right)} \prod_{i=1}^{I_{f}}\left(\theta_{f i}\right)^{v_{i}+y_{f i}^{j}-1} d \theta_{f 1} \cdots d \theta_{f I_{f}-1}
\end{aligned}
$$


which may be evaluated by:

$$
\frac{\prod_{i=1}^{I_{f}} \Gamma\left(v_{i}+y_{f i}^{j}\right)}{\Gamma\left(\sum_{i=1}^{I_{f}} v_{i}+y_{f i}^{j}\right)} \frac{1}{N} \sum_{l=1}^{N} \frac{1}{\left(\sum_{i=1}^{I_{f}}\left(\tau_{f}+\gamma_{f i}^{j}\right) \theta_{f i}^{\ell}\right)^{I_{f f f}+\sum_{i=1}^{I_{f} y_{f i}^{j}}} .}
$$

In fact, we can generate random numbers $a_{f i}^{\ell}$ from the gamma density with parameters $\left(v_{i}+y_{f i}^{j}, 1\right)$, for $i=1, \ldots, I_{f}$ and $\ell=1, \ldots, N$ where $N$ is the number of iterations in the Monte Carlo estimation. So, writing $\theta_{f i}^{\ell}=\frac{a_{f i}^{\ell}}{\sum_{i=1}^{I_{f}} a_{f i}^{\ell}}$, we obtain values of a Dirichlet $\left(v_{i}+y_{f i}^{j}, \cdots, v_{I_{f}}+y_{f I_{f}}^{j}\right)$. The accidents distribution observed at period $j$ for the $I_{f}$ vehicles of the fleet $f$ is approximately equal to:

$$
\begin{aligned}
& P\left(y_{f 1}^{j}, \cdots, y_{f I_{f}}^{j} \mid \gamma_{f 1}^{j}, \cdots, \gamma_{f I_{f}}^{j}\right) \approx \\
& \prod_{i=1}^{I_{f}}\left(\frac{\left(\gamma_{f i}^{j}\right)^{y_{f i}^{j}} \Gamma\left(v_{i}+y_{f i}^{j}\right)}{\Gamma\left(y_{f i}^{j}+1\right) \Gamma\left(v_{i}\right)}\right) \frac{\tau_{f}^{I_{f} \tau_{f}} \Gamma\left(I_{f} \tau_{f}+\sum_{i=1}^{I_{f}} y_{f i}^{j}\right)}{\Gamma\left(I_{f} \tau_{f}\right)} \frac{\Gamma\left(\sum_{i=1}^{I_{f}} v_{i}\right)}{\Gamma\left(\sum_{i=1}^{I_{f}} v_{i}+\sum_{i=1}^{I_{f}} y_{f i}^{j}\right)} \\
& \frac{1}{N} \sum_{\ell=1}^{N} \frac{1}{\left(\sum_{i=1}^{I_{f}}\left(\tau_{f}+\gamma_{f i}^{j}\right) \theta_{f i}^{\ell}\right)^{I_{f \tau f}+\sum_{i=1}^{I_{f} y j} j} .} .
\end{aligned}
$$

\subsection{Econometric estimations}

\subsubsection{Descriptive statistics}

The data come from the files of the Société d'assurance automobiles du Québec (henceforth referred to as the SAAQ), dating from 1997 to 1998 (for a detailed description of the data base see Dionne, Desjardins, and Pinquet, 1999, 2001). We had access to data on the two years from 43,679 carriers of merchandise by truck. More than two thirds of the carriers have only one vehicle. At 31 December 1997 and 31 December 1998, these small carriers owned about $30 \%$ of the 103,848 heavy trucks with authorization to circulate at least one day, so the econometric estimation was made with 73,328 trucks from 13,159 carriers. We use the 1998 data for information on accidents and characteristics of vehicles and fleets and the 1997 data for traffic violations of both the drivers (speeding, ...) 
and the fleets (overload, ...), so as to respect the SAAQ's rating policy. Moreover, this approach reduces the problem of simultaneity between the "violations" and "accidents" variables.

It should be mentioned that a vehicle was not necessarily authorized to circulate 365 days in 1998. On average, a vehicle was authorized to circulate $88.5 \%$ of 1998. Depending on the size of the fleet, this average percentage will vary between $86.7 \%$ and $93.9 \%$. To obtain an annual statistic, we calculated the number of trucks in trucks-year, by adding the number of days each truck was authorized to circulate and then dividing by 365 days. The average frequency of total accidents per truck-year is 0.1592 . This average increases as the size of the fleet increases, but decreases when the fleet contains more than 150 trucks.

\subsubsection{Estimation of parameters}

We used the maximum likelihood method to estimate the unknown parameters, $v, \tau_{f}, \beta=\left(\beta_{1}, \cdots, \beta_{m}\right)$ where $m$ is the number of observed characteristics of the truck and the fleet. We assume that each element of the vector $\left(v_{1}, v_{2}, \cdots, v_{I_{f}}\right)$ is equal to $v$. For the different methods of estimation presented in this article, we used the nonlinear optimization subroutines available in the IML procedure in SAS. The initial estimates $\beta_{0}$ of $\beta=\left(\beta_{1}, \cdots, \beta_{m}\right)$ are the maximum likelihood estimates using the negative binomial model. Initial values of parameters $v, \tau_{f}$ were set equal to one.

To maximize the Log-likelihood function of the joint-accidents probability of equation (10), the Newton-Raphson method has been used. This method needs the gradient and the Hessian matrix and, therefore, it requires first- and second-order derivatives of the objective function (see Appendix B for details). To divide the trucks into two groups, we took the maximum likelihood estimates of the negative binomial model of $\beta_{0}=\left(\beta_{1}, \cdots, \beta_{m}\right)$ to estimate $\gamma_{0 f i}=d e^{X \beta_{0}}$ for all the vehicles. Then we calculated the mean, $\bar{\gamma}=\frac{1}{N} \sum_{f=1}^{F} \sum_{i=1}^{I_{f}} \gamma_{0 f i}$, where $\mathrm{N}$ is the total number of vehicles. The truck $i$ is in group 1 if $\gamma_{0 f i} \leq \bar{\gamma}$ or it is in group 2 if $\gamma_{0 f i}>\bar{\gamma}$. The variance-covariance matrix of the asymptotic distribution is calculated from the Hessian matrix at $\hat{v}, \hat{\tau}_{f}, \hat{\beta}=\left(\hat{\beta}_{1}, \cdots, \hat{\beta}_{m}\right)$.

To maximize the Log-likelihood function of equation (11), we used the Nelder-Mead simplex method. In order to determine the variance-covariance matrix of the asymptotic distribution, we used the subroutine NLPFDD of SAS/IML to compute finite difference approximations of first-and secondorder derivatives in order to obtain the Hessian matrix at $\hat{v}, \hat{\tau}_{f}, \hat{\beta}=\left(\hat{\beta}_{1}, \cdots, \hat{\beta}_{m}\right)$.

There is quite a large volume of data; so to make a radical reduction in computation time, we calculated the Log-likelihood function, the gradient and the Hessian matrix with a homemade $\mathrm{C}$ program. We created an interface to communicate the data between the SAS program and the C program. We set up a method called "Named Pipe" for passing data to and from the SAS System. The execution speed in SAS/IML was very slow compared to the $C$ program. 
TABLE 1

PARAmeters Estimation

\begin{tabular}{|c|c|c|c|c|c|}
\hline \multirow[b]{2}{*}{ Explanatory variable } & \multicolumn{2}{|c|}{ Hypergeometric } & \multicolumn{2}{|c|}{ Monte Carlo method } & \multirow[b]{2}{*}{ Statistic $\mathbf{t}$} \\
\hline & Coefficient & $\begin{array}{l}\text { Standard } \\
\text { deviation }\end{array}$ & Coefficient & $\begin{array}{l}\text { Standard } \\
\text { deviation }\end{array}$ & \\
\hline Constant & -3.4049 & 0.0978 & -3.4416 & 0.0619 & 0,3171 \\
\hline Number of years as carrier at 31 December 1998 & -0.0596 & 0.0056 & -0.0614 & 0.0055 & 0,2293 \\
\hline $\begin{array}{l}\text { Sector of activity in } 1998 \\
\text { Other sector } \\
\text { General public trucking } \\
\text { Bulk public trucking } \\
\text { Private trucking } \\
\text { Short-term rental firm } \\
\end{array}$ & $\begin{array}{l}-0.2021 \\
0.1675 \\
\text { Referen } \\
0.0810 \\
0.6476\end{array}$ & $\begin{array}{c}0.3404 \\
0.0740 \\
\text { group } \\
0.0583 \\
0.1468\end{array}$ & $\begin{array}{l}-0.2979 \\
0.1087 \\
\text { Referen } \\
0.0381 \\
0.5576\end{array}$ & $\begin{array}{c}0.1644 \\
0.1013 \\
\text { e group } \\
0.0757 \\
0.1445\end{array}$ & $\begin{array}{l}0,2534 \\
0,4687 \\
0,4490 \\
0,4369\end{array}$ \\
\hline $\begin{array}{l}\text { Size of fleet } \\
2 \\
3 \\
4 \text { to } 5 \\
6 \text { to } 9 \\
10 \text { to } 20 \\
21 \text { to } 50 \\
\text { More than } 50\end{array}$ & $\begin{array}{l}\text { Referen } \\
0.1805 \\
0.2076 \\
0.3117 \\
0.4111 \\
0.4358 \\
0.5907\end{array}$ & $\begin{array}{l}\text { group } \\
0.0488 \\
0.0473 \\
0.0474 \\
0.0490 \\
0.0626 \\
0.0970\end{array}$ & $\begin{array}{l}\text { Referen } \\
0.1953 \\
0.2260 \\
0.3373 \\
0.4369 \\
0.4667 \\
0.5940\end{array}$ & $\begin{array}{l}\text { group } \\
0.0566 \\
0.0462 \\
0.0503 \\
0.0463 \\
0.0766 \\
0.0980\end{array}$ & $\begin{array}{l}-0,1980 \\
-0,2783 \\
-0,3704 \\
-0,3827 \\
-0,3124 \\
-0,0239\end{array}$ \\
\hline Number of days authorized to circulate in 1997 & 1.8679 & 0.0730 & 1.8818 & 0.0234 & $-0,1813$ \\
\hline $\begin{array}{l}\text { Number of violations of trucking standards in } 1997 \\
\text { For overload } \\
\text { For excessive size } \\
\text { For poorly secured cargo } \\
\text { For failure to respect service hours } \\
\text { For failure to pass mechanical inspection } \\
\text { For other reasons }\end{array}$ & $\begin{array}{l}0.1803 \\
0.6118 \\
0.4611 \\
0.2761 \\
0.3630 \\
0.3980\end{array}$ & $\begin{array}{l}0.0389 \\
0.2840 \\
0.1148 \\
0.1367 \\
0.0940 \\
0.1780\end{array}$ & $\begin{array}{l}0.1949 \\
0.6444 \\
0.4893 \\
0.3217 \\
0.4051 \\
0.3638\end{array}$ & $\begin{array}{l}0.0439 \\
0.1191 \\
0.2212 \\
0.1545 \\
0.1113 \\
0.1109\end{array}$ & $\begin{array}{r}-0,2489 \\
-0,1059 \\
-0,1132 \\
-0,2210 \\
-0,2890 \\
0,1631\end{array}$ \\
\hline $\begin{array}{l}\text { Type of vehicle use } \\
\text { Commercial use including transport of goods } \\
\text { without C.T.Q. permit } \\
\text { Transport of other than "bulk" goods } \\
\text { Transport of "bulk" goods }\end{array}$ & $\begin{array}{r}-0.1163 \\
-0.1376 \\
\text { Referen }\end{array}$ & $\begin{array}{c}0.0535 \\
0.0654 \\
\text { group }\end{array}$ & $\begin{array}{r}-0.0650 \\
-0.0778 \\
\text { Referen }\end{array}$ & $\begin{array}{r}0.0635 \\
0.0752 \\
\text { e group }\end{array}$ & $\begin{array}{l}-0,6178 \\
-0,6000\end{array}$ \\
\hline $\begin{array}{l}\text { Type of fuel } \\
\text { Diesel } \\
\text { Gas } \\
\text { Others } \\
\end{array}$ & $\begin{array}{c}\text { Referen } \\
-0.4402 \\
-0.1813\end{array}$ & $\begin{array}{l}\text { group } \\
0.0429 \\
0.1776\end{array}$ & Reference group & $\begin{array}{l}\text { e group } \\
0.0457 \\
0.1668\end{array}$ & $\begin{array}{l}0,0702 \\
0,0558\end{array}$ \\
\hline $\begin{array}{l}\text { Number of cylinders } \\
1 \text { to } 5 \text { cylinders } \\
6 \text { to } 7 \text { cylinders } \\
8 \text { or more than } 10 \text { cylinders } \\
\end{array}$ & $\begin{array}{l}0.1814 \\
0.3548 \\
\text { Referen }\end{array}$ & $\begin{array}{c}0.1025 \\
0.0353 \\
\text { group }\end{array}$ & $\begin{array}{l}0.1785 \\
0.3642 \\
\text { Referen }\end{array}$ & $\begin{array}{l}0.0974 \\
0.0378 \\
\text { e group }\end{array}$ & $\begin{array}{r}0,0205 \\
-0,1817\end{array}$ \\
\hline $\begin{array}{l}\text { Number of axles } \\
2 \text { axles }(3,000 \text { to } 4,000 \mathrm{~kg}) \\
2 \text { axles (more than } 4,000 \mathrm{~kg}) \\
3 \text { axles } \\
4 \text { axles } \\
5 \text { axles } \\
6 \text { axles or more }\end{array}$ & $\begin{array}{l}-0.3480 \\
-0.3143 \\
-0.1677 \\
-0.1442 \\
-0.1913 \\
\text { Referen }\end{array}$ & $\begin{array}{l}0.0529 \\
0.0370 \\
0.0361 \\
0.0488 \\
0.0424 \\
\text { group }\end{array}$ & $\begin{array}{l}-0.3298 \\
-0.3017 \\
-0.1676 \\
-0.1474 \\
-0.1967 \\
\text { Referen }\end{array}$ & $\begin{array}{l}0.0573 \\
0.0393 \\
0.0385 \\
0.0477 \\
0.0433 \\
\text { e group }\end{array}$ & $\begin{array}{r}-0,2334 \\
-0,2334 \\
-0,0019 \\
0,0469 \\
0,0891\end{array}$ \\
\hline
\end{tabular}




\begin{tabular}{|c|c|c|c|c|c|}
\hline \multirow[b]{2}{*}{ Explanatory variables } & \multicolumn{2}{|c|}{ Two groups method } & \multicolumn{2}{|c|}{ Monte Carlo method } & \multirow[b]{2}{*}{ Statistic $\mathrm{t}$} \\
\hline & Coefficient & $\begin{array}{l}\text { Standard } \\
\text { deviation }\end{array}$ & Coefficient & $\begin{array}{l}\text { Standard } \\
\text { deviation }\end{array}$ & \\
\hline \multicolumn{6}{|c|}{ Number of violations with demerit points in 1997} \\
\hline For speeding & 0.2648 & 0.0241 & 0.2810 & 0.0290 & $-0,4296$ \\
\hline For driving under suspension & 0.4725 & 0.1356 & 0.4271 & 0.1621 & 0,2148 \\
\hline For running a red light & 0.4031 & 0.0653 & 0.4570 & 0.0875 & $-0,4937$ \\
\hline For ignoring stop sign or traffic agent & 0.5134 & 0.0684 & 0.5134 & 0.0703 & 0,0000 \\
\hline For not wearing a seat belt & 0.1741 & 0.1059 & 0.1877 & 0.1088 & $-0,0896$ \\
\hline For other offenses & 1.1218 & 0.0769 & 1.2257 & 0.1371 & $-0,6610$ \\
\hline$\hat{\hat{v}}$ & 2.2056 & 0.2098 & 2.2710 & 0.2179 & $-0,2162$ \\
\hline$\hat{\tau}^{-1}$ (fleets of 2 trucks) & 1.5615 & 0.2686 & 1.5107 & 0.2748 & 0,1322 \\
\hline$\hat{\tau}^{-1}$ (fleets of 3 trucks) & 2.1061 & 0.3494 & 2.0658 & 0.4532 & 0,0704 \\
\hline$\hat{\tau}^{-1}$ (fleets of 4 to 5 trucks) & 3.0853 & 0.4048 & 3.0481 & 0.3961 & 0,0657 \\
\hline$\hat{\tau}^{-1}$ (fleets of 6 to 9 trucks) & 3.5167 & 0.4661 & 3.4933 & 0.4159 & 0,0375 \\
\hline$\hat{\tau}^{-1}$ (fleets of 10 to 20 trucks) & 6.4867 & 0.7011 & 6.4552 & 0.6521 & 0,0329 \\
\hline$\hat{\tau}^{-1}$ (fleets of 21 to 50 trucks) & 15.9511 & 1.9657 & 15.6209 & 4.3179 & 0,0696 \\
\hline$\hat{\tau}^{-1}$ (fleets more than 50 trucks) & 118.4366 & 15.7770 & 89.0506 & 10.0112 & 1,5727 \\
\hline Log-likelihood & $\begin{array}{l}-30,494 \\
\end{array}$ & & $-30,500$ & & \\
\hline Number of carriers & 13,159 & & 13,159 & & \\
\hline Number of vehicles & 73,328 & & 73,328 & & \\
\hline
\end{tabular}

We obtained a speed of 20 to 30 times faster with the C program. In addition, we increased the execution speed by adding many machines in parallel since each machine can make the calculations independently.

The results for all fleet sizes with 2 vehicles and more are presented in Table 1 for the two methods of estimation. We consider first the hypergeometric approximation. For fleets with more than two trucks, we divided the trucks into two groups, as explained above. For fleets with two vehicles, we estimated the exact model of Section 2.1.2. We used the 10\% threshold ( $p$-value lower than or equal to 0.10 ) to consider a statistical coefficient different from zero.

We note in Table 1 that the vehicles with more experience (number of years as carrier) have fewer accidents. The results also indicate that the factors explaining accidents include: the carrier's size and sector of activity; the type of use to which the vehicle was put; the type of fuel; the number of cylinders; and the number of axles. Vehicles with fleet violations (violations of trucking standards) in 1997 are more at risk for accidents in 1998 than those without these types of offenses. Moreover, vehicles whose drivers have accumulated violations in 1997 represent higher risks for accidents in 1998 than those without such violations.

Table 1 also reports the results on the parameters for random effects distributions. Regression indicates that the seven $\tau_{f}$ parameters are significant, which means that we can reject the Poisson distribution and apply a bonus malus insurance rating model to these fleets. It is important to mention that we estimated seven parameters because these parameters are affected by fleet size. The $v$ parameter is also significant at $p<0.0001$. This coefficient is not 
affected by fleet size. These results signal that both vehicle and fleet effects can be used in calculating premiums.

The Monte-Carlo-method results are very similar to those of the hypergeometric method, with few exceptions. The last column of the table indicates the $t$-statistic for the parametric difference between the two models. The only difference in the parameters concerns the $\tau_{f}^{-1}$ coefficient for fleets with more than 50 vehicles, but this difference is not statistically significant. The values of the Log-likelihood are also very similar.

The $\beta$ coefficients will be very useful in estimating a priori risks when calculating insurance premiums, whereas coefficients $\tau_{f}$ and $v$ will be useful in adjusting premiums to fit the past accident records of vehicles and fleets in the bonus malus model.

An important research step consists in verifying the effectiveness of the proposed method in comparison with other models in the literature. Table 2

TABLE 2

Descriptive Statistics From Four Estimated Models

\begin{tabular}{l|c|c|c|c}
\hline \hline Statistics & Poisson & $\begin{array}{c}\text { Negative } \\
\text { binomial }\end{array}$ & $\begin{array}{c}\text { Hypergeometric } \\
\text { method }\end{array}$ & $\begin{array}{c}\text { Monte Carlo } \\
\text { method }\end{array}$ \\
\hline Residuals & -0.1154 & -0.1163 & -0.0889 & -0.0889 \\
\hline MEDIAN & 0.1316 & 0.1343 & 0.1040 & 0.1040 \\
\hline IQR & 0.0659 & 0.0671 & 0.0515 & 0.0517 \\
\hline MAD & 0.2399 & 0.2415 & 0.2046 & 0.2050 \\
\hline MAE & 0.4083 & 0.4102 & 0.3523 & 0.3508 \\
\hline STD & $-31,547$ & $-31,217$ & $-30,497$ & $-30,500$ \\
\hline Goodness-of-fit & 63,497 & 62,849 & 61,487 & 61,493 \\
\hline Log L & 63,166 & 62,508 & 61,082 & 61,088 \\
\hline BIC & 36 & 37 & 44 & 44 \\
\hline AIC & 35 seconds & 55 seconds & 15 minutes & 1 month \\
\hline Number of parameters $(k)$ & $(1 \mathrm{CPU})$ & $(1 \mathrm{CPU})$ & $(1 \mathrm{CPU})$ & $(35$ CPUs $)$ \\
\hline Computation time &
\end{tabular}

Bayesian Information Criterion $(\mathrm{BIC})=-2 \ln L+k \ln (N)$; Akaikes Information Criterion (AIC) $=2 \ln L+2 k$; where $k$ and $N$ are the number of parameters and observations respectively.

Q1: $25^{\text {th }}$ percentile; Q2 (or median): $50^{\text {th }}$ percentile; Q3: $75^{\text {th }}$ percentile.

IQR: Interquartile range: $\mathrm{Q}_{3}-\mathrm{Q}_{1}$.

MAD: Median Absolute Deviation: $\mathrm{MAD}=\operatorname{MEDIAN}_{1 \leq i \leq n}\left\{\left|e_{i}-Q_{2}\right|\right\}$ where $e_{i}=y_{f i}^{j}-E\left(\lambda_{f i}^{j} \mid y_{f 1}^{j} \cdots y_{f I_{f}}^{j}\right)$. MAE: Mean Absolute Error: $\quad \mathrm{MAE}=n^{-1} \sum_{i=1}^{n}\left|e_{i}\right|$. 
presents different statistics for four models: the Poisson model, the negative binomial model, the hypergeometric method, and the Monte Carlo method. The first two models (Poisson and negative binomial) do not have fleet effects, while both the hypergeometric method and the Monte Carlo method contain a fleet effect. The results clearly show that the hypergeometric method performs very well. All the statistics are very close to those of the Monte Carlo method. We now compare these two models with the two models containing no fleet effect. The first five statistics are for residuals. All the statistics related to the residuals go in the right direction. The three other statistics are for goodness-of-fit. Again, the hypergeometric model performs very well. It should be mentioned that the computation time of the hypergeometric approximation can be reduced significantly by using more CPU. We present the results with 1 CPU to compare them with the Poisson and the negative binomial models. With 4 CPU, the estimation time with the hypergeometric method is 82 seconds.

\subsubsection{Predictive probability}

One interesting feature of the Bayesian parametric model is it allows us to compute directly the predictive probabilities of accidents. We propose an example for a fleet of two vehicles.

Let $y_{f 1}^{t+1}$ be the number of accidents of truck 1 of fleet $f$ at time $t+1$,

$y_{f 2}^{t+1}$ be the number of accidents of truck 2 of fleet $f$ at time $t+1$,

$y_{f 1}^{t}$ be the number of accidents of truck 1 of fleet $f$ at time $t$,

and $y_{f 2}^{t}$ be the number of accidents of truck 2 of fleet $f$ at time $t$.

Given the previous data, it can be shown that the predictive probability of $\left(y_{f 1}^{t+1}\right.$, $y_{f 2}^{t+1}$ ) can be written as:

$$
P\left(y_{f 1}^{t+1}, y_{f 2}^{t+1} \mid y_{f 1}^{t}, y_{f 2}^{t}, \gamma_{f 1}^{t}, \gamma_{f 2}^{t}\right)=\frac{P\left(y_{f 1}^{t}, y_{f 2}^{t}, y_{f 1}^{t+1}, y_{f 2}^{t+1} \mid \gamma_{f 1}^{t}, \gamma_{f 2}^{t}\right)}{P\left(y_{f 1}^{t}, y_{f 2}^{t} \mid \gamma_{f 1}^{t}, \gamma_{f 2}^{t}\right)},
$$

where

$$
\begin{gathered}
P\left(y_{f 1}^{t}, y_{f 2}^{t}, y_{f 1}^{t+1}, y_{f 2}^{t+1} \mid \gamma_{f 1}^{t}, \gamma_{f 2}^{t}\right)=\iint \frac{\left(\gamma_{f 1}^{t}\right)^{y_{f 1}^{t}+y_{f}^{t+1}}\left(\gamma_{f 2}^{t}\right)^{y_{j 2}^{t}+y_{\rho 2}^{t+1}} \theta_{f}^{y_{f 1}^{t}+y_{f 1}^{t+1}}\left(1-\theta_{f}\right)^{y_{f 2}^{t}+y_{f 2}^{t+1}}}{\Gamma\left(y_{f 1}^{t}+1\right) \Gamma\left(y_{f 2}^{t}+1\right) \Gamma\left(y_{f 1}^{t+1}+1\right) \Gamma\left(y_{f 2}^{t+1}+1\right)} \frac{\tau_{f}^{2 \tau_{f}}}{\Gamma\left(2 \tau_{f}^{\tau_{f}}\right)} \\
\times \alpha^{2 \tau_{f}+\sum_{i=1}^{2} \sum_{f i}^{t}+\sum_{i=1}^{t} y_{f i}^{t+1}-1} e^{-\alpha\left[\tau_{f}+2 \theta_{f} \gamma_{f 1}^{t}+2\left(1-\theta_{f}\right) \gamma_{f 2}^{t}\right]} \frac{\Gamma\left(\sum_{i=1}^{2} v_{i}\right)}{\Gamma\left(v_{1}\right) \Gamma\left(v_{2}\right)} \theta_{f}^{v_{1}}\left(1-\theta_{f}\right)^{v_{2}} d \alpha d \theta_{f}
\end{gathered}
$$




$$
\begin{aligned}
& =\frac{\left(\gamma_{f 1}^{t}\right)^{y_{f 1}^{t}+y_{f 1}^{t+1}}\left(\gamma_{f 2}^{t}\right)^{y_{f 2}^{t}+y_{f 2}^{t+1}}}{\Gamma\left(y_{f 1}^{t}+1\right) \Gamma\left(y_{f 2}^{t}+1\right) \Gamma\left(y_{f 1}^{t+1}+1\right) \Gamma\left(y_{f 2}^{t+1}+1\right)} \frac{\tau_{f}^{2 \tau_{f}}}{\Gamma\left(2 \tau_{f}^{\tau_{f}}\right)} \frac{\Gamma\left(\sum_{i=1}^{2} v_{i}\right)}{\Gamma\left(v_{1}\right) \Gamma\left(v_{2}\right)} \\
& \times \frac{\Gamma\left(2 \tau_{f}+\sum_{i=1}^{2} y_{f i}^{t}+\sum_{i=1}^{2} y_{f i}^{t+1}\right)}{\left(\tau_{f}+2 \gamma_{f 2}^{t}\right)^{2 \tau_{f}+\sum_{i=1}^{2} y_{f i}^{t}+\sum_{i=1}^{2} y_{f i}^{t+1}}} \frac{\Gamma\left(v_{1}+y_{f 1}^{t}+y_{f 1}^{t+1}\right) \Gamma\left(v_{2}+y_{f 2}^{t}+y_{f 2}^{t+1}\right)}{\Gamma\left(\sum_{i=1}^{2} v_{i}+\sum_{i=1}^{2} y_{f i}^{t}+\sum_{i=1}^{2} y_{f i}^{t+1}\right)} \\
& \times{ }_{2} F_{1}\left(v_{1}+y_{f 1}^{t}+y_{f 1}^{t+1} ; 2 \tau_{f}+\sum_{i=1}^{2} y_{f i}^{t}+\sum_{i=1}^{2} y_{f i}^{t+1} ; \sum_{i=1}^{2} v_{i}+\sum_{i=1}^{2} y_{f i}^{t}+\sum_{i=1}^{2} y_{f i}^{t+1} ; 2\left(\frac{\gamma_{f 2}^{t}-\gamma_{f 1}^{t}}{\tau_{f}+2 \gamma_{f 2}^{t}}\right)\right) \text {. }
\end{aligned}
$$

Hence substituting the above value in (12), the predictive probability is given by

$$
\begin{aligned}
& P\left(y_{f 1}^{t+1}, y_{f 2}^{t+1} \mid y_{f 1}^{t}, y_{f 2}^{t}, \gamma_{f 1}^{t}, \gamma_{f 2}^{t}\right)= \\
& \frac{\left(\gamma_{f 1}^{t}\right)^{y_{f 1}^{t+1}}\left(\gamma_{f 2}^{t}\right)^{y_{f 2}^{t+1}}}{\Gamma\left(y_{f 1}^{t+1}+1\right) \Gamma\left(y_{f 2}^{t+1}+1\right)} \frac{\Gamma\left(2 \tau_{f}+\sum_{i=1}^{2} y_{f i}^{t}+\sum_{i=1}^{2} y_{f i}^{t+1}\right)}{\Gamma\left(2 \tau_{f}+\sum_{i=1}^{2} y_{f i}^{t}\right)} \frac{\left(\tau_{f}+\gamma_{f 2}^{t}\right)^{2 \tau_{f}+\sum_{i=1}^{2} y_{f i}^{t}}}{\left(\tau_{f}+2 \gamma_{f 2}^{t}\right)^{2 \tau_{f}+\sum_{i=1}^{2} y_{f i}^{t}+\sum_{i=1}^{2} y_{f i}^{t+1}}} \\
& \times \frac{\Gamma\left(v_{1}+y_{f 1}^{t}+y_{f 1}^{t+1}\right) \Gamma\left(v_{2}+y_{f 2}^{t}+y_{f 2}^{t+1}\right)}{\Gamma\left(v_{1}+y_{f 1}^{t}\right) \Gamma\left(v_{2}+y_{f 2}^{t}\right)} \frac{\Gamma\left(\sum_{i=1}^{2} v_{i}+\sum_{i=1}^{2} y_{f i}^{t}\right)}{\Gamma\left(\sum_{i=1}^{2} v_{i}+\sum_{i=1}^{2} y_{f i}^{t}+\sum_{i=1}^{2} y_{f i}^{t+1}\right)} \\
& \quad \times \frac{{ }_{2} F_{1}\left(v_{1}+y_{f 1}^{t}+y_{f 1}^{t+1} ; 2 \tau_{f}+\sum_{i=1}^{2} y_{f i}^{t}+\sum_{i=1}^{2} y_{f i}^{t+1} ; \sum_{i=1}^{2} v_{i}+\sum_{i=1}^{2} y_{f i}^{t}+\sum_{i=1}^{2} y_{f i}^{t+1} ; 2\left(\frac{\gamma_{f 2}^{t}-\gamma_{f 1}^{t}}{\tau_{f}+2 \gamma_{f 2}^{t}}\right)\right)}{{ }_{2} F_{1}\left(v_{1}+y_{f 1}^{t} ; 2 \tau_{f}+\sum_{i=1}^{2} y_{f i}^{t} ; \sum_{i=1}^{2} v_{i}+\sum_{i=1}^{2} y_{f i}^{t} ;\left(\frac{\gamma_{f 2}^{t}-\gamma_{f 1}^{t}}{\tau_{f}+\gamma_{f 2}^{t}}\right)\right)} .
\end{aligned}
$$

Table 2A presents examples of predictive probabilities for a fleet of two vehicles. For example, if the fleet had no accident during the previous year $(t)$, its probability of having one accident in the current year $(t+1)$ is $9.81 \%$ with the second truck and $6.44 \%$ with the first one. If the second truck had an accident during the previous year, the corresponding probabilities increase to $16.98 \%$ and $7.78 \%$ respectively. As we shall see in the next section, these probabilities can be used to obtain the equivalent of a Bonus Malus Factor (BMF) for small fleets. However, the method with predictive probabilities would rapidly become very complex with large fleets. 
TABLE 2A

Predictive Probabilities for Fleets of Two Trucks

\begin{tabular}{c|c|c|c|c|c|c|c}
\hline \hline \multirow{2}{*}{$y_{f 1}^{t+1}$} & \multirow{2}{*}{$y_{f 2}^{t+1}$} & \multicolumn{3}{|c|}{$P\left(y_{f 1}^{t+1}, y_{f 2}^{t+1} \mid y_{1}^{t}, y_{2}^{t}, \gamma_{f 1}^{t}, \gamma_{f 2}^{t}\right)$ with $\hat{\tau}_{f}=0.6404 ; \hat{v}=2.2056$ and $\gamma_{f 1}^{t}=0.114 ; \gamma_{f 2}^{t}=0.185$} \\
\cline { 3 - 8 } & & $y_{f 1}^{t}=0 ; y_{f 2}^{t}=0$ & $y_{f 1}^{t}=1 ; y_{f 2}^{t}=0$ & $y_{f 1}^{t}=0 ; y_{f 2}^{t}=1$ & $y_{f 1}^{t}=2 ; y_{f 2}^{t}=0$ & $y_{f 1}^{t}=1 ; y_{f 2}^{t}=1$ & $y_{f 1}^{t}=0 ; y_{f 2}^{t}=2$ \\
\hline 0 & 0 & 0.8017 & 0.6838 & 0.6663 & 0.5866 & 0.5683 & 0.5508 \\
0 & 1 & 0.0981 & 0.1215 & 0.1698 & 0.1263 & 0.1761 & 0.2218 \\
0 & 2 & 0.0125 & 0.0188 & 0.0342 & 0.0221 & 0.0401 & 0.0621 \\
1 & 0 & 0.0644 & 0.1176 & 0.0778 & 0.1621 & 0.1185 & 0.0782 \\
1 & 1 & 0.0115 & 0.0253 & 0.0241 & 0.0393 & 0.0414 & 0.0356 \\
1 & 2 & 0.0018 & 0.0044 & 0.0055 & 0.0075 & 0.0103 & 0.0109 \\
2 & 0 & 0.0055 & 0.0163 & 0.0081 & 0.0315 & 0.0184 & 0.0092 \\
2 & 1 & 0.0012 & 0.0039 & 0.0028 & 0.0083 & 0.0070 & 0.0046 \\
2 & 2 & 0.0002 & 0.0007 & 0.0007 & 0.0017 & 0.0018 & 0.0015 \\
\hline \hline
\end{tabular}

We now present our extended bonus malus system which includes information on non-observable heterogeneity.

\section{BonUs MALUS}

\subsection{Optimal bonus malus system}

To construct an optimal bonus malus system (Lemaire, 1985; Dionne and Vanasse, 1989, 1992) based on the number of past accidents recorded for a truck as well as those observed for its fleet, we must calculate the premium for a truck of a given fleet at period $t+1$ using the following mathematical expectation relation:

$$
\gamma_{f i}^{t+1}\left(\frac{E\left(\theta_{f i} \alpha_{f} \mid y_{f}, X_{f}\right)}{E\left(\theta_{f i} \alpha_{f}\right)}\right),
$$

where $y_{f}$ and $X_{f}$ measure past accidents of vehicles in fleet $f$ up to period $t$ and past and current characteristics of all the trucks in fleet $f$ up to $t+1$. It is important to observe that $\gamma_{f i}^{t+1}$ is only a function of the observable characteristics in period $t+1$, while $y_{f}$ and $X_{f}$ take into account the all past and current information available (Dionne and Vanasse, 1989).

The term $\gamma_{f i}^{t+1}$ corresponds to the part of the mathematical expectation obtained from the econometric regressions. It is equal to $d_{f i}^{t+1} e^{X_{f i}^{t+1} \beta}$ where $d_{f i}^{t+1}$ is the number of days that vehicle $i$ of fleet $f$ is authorized to circulate in period $t+1$ divided by the total number of days in period $t+1$. As already indicated, this variable measures exposure to risk. The regression component corresponds to $X_{f i}^{t+1} \beta$ where the vector of coefficients $(\beta)$ is estimated by means of econo- 
metric models and $X_{f i}^{t+1}=\left(x_{f i 1}^{t+1}, \cdots, x_{f i m}^{t+1}\right)$ represents the observable $m$ characteristics of truck $i$ in fleet $f$ at the beginning of period $t+1 . X_{f}=\left(X_{f 1}^{1}, \cdots, X_{f I_{f}}^{1}, \cdots\right.$, $X_{f 1}^{t+1}, \cdots, X_{f I_{f}}^{t+1}$ ) gives the $m$ characteristics of all the trucks in fleet $f$ up to the $t+1$ period. The vector $y_{f}=\left(y_{f 1}^{1}, \cdots, y_{f I_{f}}^{1}, \cdots, y_{f 1}^{t}, \cdots, y_{f f_{f}}^{t}\right)$ represents the accidents of vehicles in fleet $f$ up to period $t$ and $E\left(\theta_{f i} \alpha_{f} \mid y_{f}, X_{f}\right)$ designates the mathematical expectation of the fleet and vehicle effects attributable to vehicle $i$, based on past experience as measured by accidents accumulated over the preceding $t$ periods and characteristics over the $t+1$ periods. As we shall see, the modeling proposed will take into account both the accidents of vehicle $i$ and those of its fleet $f$. These effects account for the unobservable factors which can affect the accidents of trucks and fleets: $\alpha_{f}$ is the effect associated with fleet $f$ and $\theta_{f i}$ is the weight truck $i$ in fleet $f$ actually exerts on this fleet effect. Finally, $E\left(\theta_{f i} \alpha_{f}\right)$ gives the mathematical expectation of the two effects attributable to truck $i$ not conditional on past experience. The last term is used to normalize the $\mathrm{BMF}$ at 1 when the insurer has no experience with a particular vehicle.

The preceding equation comes from a Bayesian analysis of the evolution of accidents over time. We are now going to show its explicit form under the hypotheses of statistical distribution for the two random effects. We know that the true mathematical expectation of the number of accidents for truck $i$ of fleet $f$ at period $t+1$ is equal to $\lambda_{f i}^{t+1}$. It is a function of the vector for the observable characteristics of the vehicle up to period $j$ and of the random factors for fleet $\alpha_{f}$ and vehicle $\theta_{f i}$, which are supposed to be independent of time.

Given the observations obtained up to period $t+1$, the optimal estimator of this true mathematical expectation at period $t+1, \hat{\lambda}_{f i}^{t+1}\left(y_{f}, X_{f}\right)$, can be calculated as follows:

$$
\begin{aligned}
\hat{\lambda}_{f i}^{t+1}\left(y_{f}, X_{f}\right) & =\gamma_{f i}^{t+1}\left(\frac{E\left(\alpha_{f} \theta_{f i} \mid y_{f}, X_{f}\right)}{E\left(\alpha_{f}\right) E\left(\theta_{f i}\right)}\right) \\
& =\gamma_{f i}^{t+1}\left(\frac{E\left(\theta_{f i} E\left(\alpha_{f} \mid \theta_{f 1}, \cdots, \theta_{f I_{f}}, y_{f}, X_{f}\right) \mid y_{f}, X_{f}\right)}{E\left(\alpha_{f}\right) E\left(\theta_{f i}\right)}\right) .
\end{aligned}
$$

We know that:

$$
\begin{aligned}
& E\left(\theta_{f i} E\left(\alpha_{f} \mid \theta_{f 1}, \cdots, \theta_{f I_{f}}, y_{f}, X_{f}\right) \mid y_{f}, X_{f}\right)= \\
& \int_{\sum_{i=1}^{I_{f}} \theta_{f i}=1} \cdots \int \theta_{f i} E\left(\alpha_{f} \mid \theta_{f 1}, \cdots, \theta_{f I_{f}}, y_{f}, X_{f}\right) f\left(\theta_{f 1}, \cdots, \theta_{f I_{f}} \mid y_{f}, X_{f}\right) d \theta_{f 1} \cdots d \theta_{f I_{f}-1}
\end{aligned}
$$

with:

$$
f\left(\theta_{f 1}, \cdots, \theta_{f I_{f}} \mid y_{f}, X_{f}\right)=\frac{P\left(y_{f} \mid \theta_{f 1}, \cdots, \theta_{f I_{f}}, X_{f}\right) f\left(\theta_{f 1}, \cdots, \theta_{f I_{f}}\right)}{\int_{\sum_{i=1}^{t f_{f i}=1}} \cdots \int P\left(y_{f} \mid \theta_{f 1}, \cdots, \theta_{f I_{f}}, X_{f}\right) f\left(\theta_{f 1}, \cdots, \theta_{f I_{f}}\right) d \theta_{f 1} \cdots d \theta_{f I_{f}-1}} .
$$


Similarly, we can calculate:

$$
E\left(\alpha_{f} \mid \theta_{f 1}, \cdots, \theta_{f I_{f}}, y_{f}, X_{f}\right)=\int_{0}^{\infty} \alpha_{f} f\left(\alpha_{f} \mid \theta_{f 1}, \cdots, \theta_{f I_{f}}, y_{f}, X_{f}\right) d \alpha_{f}
$$

with:

$$
f\left(\alpha_{f} \mid \theta_{f 1}, \cdots, \theta_{f I_{f}}, y_{f}, X_{f}\right)=\frac{P\left(y_{f} \mid \alpha_{f}, \theta_{f 1}, \cdots, \theta_{f I_{f}}, X_{f}\right) f\left(\alpha_{f}\right)}{\int_{0}^{\infty} P\left(y_{f} \mid \alpha_{f}, \theta_{f 1}, \cdots, \theta_{f I_{f}}, X_{f}\right) f\left(\alpha_{f}\right) d \alpha_{f}} .
$$

Now let's see how we can apply this Bayesian rating formula to carriers of different sizes.

\subsubsection{Size-1 carrier}

In this situation, the conditional accident probability for the fleet is given by:

$$
\left.P\left(y_{f} \mid \alpha_{f}, X_{f}\right)=\prod_{j=1}^{t}\left[\frac{\left(\gamma_{f 1}^{j} \alpha_{f}\right)^{y_{f 1}^{j}} e^{-\gamma_{f 1}^{j} \alpha_{f}}}{\Gamma\left(y_{f 1}^{j}+1\right)}\right]=\left[\prod_{j=1}^{t} \frac{\left(\gamma_{f 1}^{j}\right)^{y_{f 1}^{j}}}{\Gamma\left(y_{f 1}^{j}+1\right)}\right]\left(\alpha_{f}\right)^{\sum_{j=1}^{t} y_{f 1}^{j}} e^{-\left(\alpha_{f} \sum_{j=1}^{t} \gamma_{f 1}^{j}\right.}\right)
$$

where $y_{f}$ measures the accidents of fleet $f$ up to $t$. It is implicitly assumed that the $y_{f i}^{j}$ are conditionally independent, which can be considered as a restrictive assumption, particularly for small fleets whose trucks are often driven by the same drivers. Given past accidents observed up to period $t$, the mathematical expectation estimator of the number of accidents for the truck in a size-one fleet at period $t+1$ is equal to:

$$
\gamma_{f 1}^{t+1} \frac{E\left(\alpha_{f} \mid y_{f}, X_{f}\right)}{E\left(\alpha_{f}\right)}=\gamma_{f 1}^{t+1}\left[\frac{\tau_{f}+\sum_{j=1}^{t} y_{f 1}^{j}}{\tau_{f}+\sum_{j=1}^{t} \gamma_{f 1}^{j}}\right] .
$$

This is the posterior gamma, a formula often used in the literature (Lemaire, 1985; Dionne and Vanasse, 1989, 1992) for individual vehicles. It does not have to account for the vehicle effect since the vehicle is the fleet.

\subsubsection{Carrier with 2 vehicles}

In this situation, the conditional accident probability for the fleet is given by: 


$$
\begin{aligned}
& P\left(y_{f} \mid \theta_{f}, \alpha_{f}, X_{f}\right)= \\
& {\left[\prod_{i=1}^{I_{f}} \prod_{j=1}^{t} \frac{\left(\gamma_{f i}^{j}\right)^{y_{j i}^{j}}}{\Gamma\left(y_{f i}^{j}+1\right)}\right]\left(\theta_{f}\right)^{\sum_{j=1}^{t} y_{f 1}^{j}}\left(1-\theta_{f}\right)^{\sum_{j=1}^{t} y_{f 2}^{j}}\left[\left(\alpha_{f}\right)^{\sum_{i=1}^{I_{f}} \sum_{j=1}^{t} y_{f i}^{j}}\right] e^{-\alpha_{f}\left(\theta_{f} \sum_{j=1}^{t} \gamma_{f_{1}^{\prime}}^{j}+\left(1-\theta_{f}\right) \sum_{j=1}^{t} \gamma_{f 2}^{j}\right),}}
\end{aligned}
$$

where $y_{f}$ measures the accidents of vehicles in fleet $f$ up to $t$.

We know that, given the past accidents observed up to period $t$ and due to the values assigned to the random effects of the 2 trucks in fleet $f$, the a posteriori density function for $\alpha_{f}$ corresponds to a gamma density with parameters:

$$
\left(2 \tau_{f}+\sum_{i=1}^{2} \sum_{j=1}^{t} y_{f i}^{j}, \tau_{f}+\theta_{f} \sum_{j=1}^{t} \gamma_{f 1}^{j}+\left(1-\theta_{f}\right) \sum_{j=1}^{t} \gamma_{f 2}^{j}\right)
$$

where $\sum_{i=1}^{2} \sum_{j=1}^{t} y_{f i}^{j}$ and $\theta_{f} \sum_{j=1}^{t} \gamma_{f 1}^{j}+\left(1-\theta_{f}\right) \sum_{j=1}^{t} \gamma_{f 2}^{j}$ take into account the accumulated experience at $t$.

So:

$$
\begin{aligned}
& f\left(\alpha_{f} \mid y_{f}, X_{f}, \theta_{f}\right)= \\
& \frac{\left(\tau_{f}+\theta_{f} \sum_{j=1}^{t} \gamma_{f 1}^{j}+\left(1-\theta_{f}\right) \sum_{j=1}^{t} \gamma_{f 2}^{j}\right)^{2 \tau_{f}+\sum_{i=1}^{2} \sum_{j=1}^{t} y_{f i}^{j}}}{\Gamma\left(2 \tau_{f}+\sum_{i=1}^{2} \sum_{j=1}^{t} y_{f i}^{j}\right)}\left(\alpha_{f}\right)^{2 \tau_{f}+\sum_{i=1}^{2} \sum_{j=1}^{j} y_{j}^{j}-1}\left[e^{-\alpha_{f}\left(\tau_{f}+\theta_{f} \sum_{j=1}^{t} \gamma_{f}^{j}+\left(1-\theta_{f}\right) \sum_{j=1}^{t} \gamma_{f^{j}}\right)}\right] .
\end{aligned}
$$

Given the past accidents observed up to period $t$ and due to the values assigned to the random effects of the 2 trucks in fleet $f$, the mathematical expectation of $\alpha_{f}$ is equal to:

$$
E\left(\alpha_{f} \mid \theta_{f}, y_{f}, X_{f}\right)=\frac{2 \tau_{f}+\sum_{i=1}^{2} \sum_{j=1}^{t} y_{f i}^{j}}{\tau_{f}+\theta_{f} \sum_{j=1}^{t} \gamma_{f 1}^{j}+\left(1-\theta_{f}\right) \sum_{j=1}^{t} \gamma_{f 2}^{j}} .
$$

Given the past accidents observed up to period $t$ for the two trucks of fleet $f$, the density function of $\theta_{f}$ is equal to:

$$
f\left(\theta_{f} \mid y_{f}, X_{f}\right)=D \frac{\left[\left(\theta_{f}\right)^{v_{1}+\sum_{j=1}^{t} y_{f 1}^{j}-1}\left(1-\theta_{f}\right)^{v_{2}+\sum_{j=1}^{t} y_{f 2}^{j}-1}\right]}{\left(\tau_{f}+\theta_{f} \sum_{j=1}^{t} \gamma_{f 1}^{j}+\left(1-\theta_{f}\right) \sum_{j=1}^{t} \gamma_{f 2}^{j}\right)^{2 \tau_{f}+\sum_{i=1}^{2} \sum_{j=1}^{t} y_{j}^{j}}}
$$


where

$$
\begin{aligned}
D^{-1}= & \frac{\prod_{i=1}^{2} \Gamma\left(v_{i}+\sum_{j=1}^{t} y_{f i}^{j}\right)}{\Gamma\left(\sum_{i=1}^{2} v_{i}+\sum_{i=1}^{2} \sum_{j=1}^{t} y_{f i}^{j}\right)\left(\tau_{f}+\sum_{j=1}^{t} \gamma_{f 2}^{j}\right)^{2 \tau_{f}+\sum_{i=1}^{2} \sum_{j=1}^{t} y_{f i}^{j}}} \\
& \times{ }_{2} F_{1}\left(v_{i}+\sum_{j=1}^{t} y_{f 1}^{j} ; 2 \tau_{f}+\sum_{i=1}^{2} \sum_{j=1}^{t} y_{f i}^{j} ; \sum_{i=1}^{2} v_{i}+\sum_{i=1}^{2} \sum_{j=1}^{t} y_{f i}^{j} ; \frac{\sum_{j=1}^{t} \gamma_{f 2}^{j}-\sum_{j=1}^{t} \gamma_{f 1}^{j}}{\tau_{f}+\sum_{j=1}^{t} \gamma_{f 2}^{j}}\right) .
\end{aligned}
$$

Given the past accidents observed up to period $t$ for the two trucks of fleet $f$, the mathematical expectation estimator of the number of accidents for truck $i$ in fleet $f$ at period $t+1$ is thus equal to:

$\gamma_{f i}^{t+1} E\left(\alpha_{f} \theta_{f i} \mid y_{f}, X_{f}\right)=\gamma_{f i}^{t+1}\left(2 \tau_{f}+\sum_{i=1}^{2} \sum_{j=1}^{t} y_{f i}^{j}\right) E\left(\frac{\theta_{f i}}{\tau_{f}+\theta_{f} \sum_{j=1}^{t} \gamma_{f 1}^{j}+\left(1-\theta_{f}\right) \sum_{j=1}^{t} \gamma_{f 2}^{j}} \mid y_{f}, X_{f}\right)$

with $\theta_{f i}=\theta_{f}$ if $i=1$ and $1-\theta_{f}$ if $i=2$.

It remains to calculate the expression:

$$
\begin{gathered}
E\left(\frac{\theta_{f i}}{\tau_{f}+\theta_{f} \sum_{j=1}^{t} \gamma_{f 1}^{j}+\left(1-\theta_{f}\right) \sum_{j=1}^{t} \gamma_{f 2}^{j}} \mid y_{f}, X_{f}\right)= \\
\int_{0}^{1} \frac{\theta_{f i}}{\tau_{f}+\theta_{f} \sum_{j=1}^{t} \gamma_{f 1}^{j}+\left(1-\theta_{f}\right) \sum_{j=1}^{t} \gamma_{f 2}^{j}} f\left(\theta_{f} \mid y_{f}, X_{f}\right) d \theta_{f} .
\end{gathered}
$$

By replacing $f\left(\theta_{f} \mid y_{f}, X_{f}\right)$ with its value given in (15), we obtain that:

$$
\begin{gathered}
E\left(\frac{\theta_{f i}}{\tau_{f}+\theta_{f} \sum_{j=1}^{t} \gamma_{f 1}^{j}+\left(1-\theta_{f}\right) \sum_{j=1}^{t} \gamma_{f 2}^{j} \mid} y_{f}, X_{f}\right)= \\
D \int_{0}^{1} \frac{\theta_{f i}\left[\left(\theta_{f}\right)^{v_{1}+\sum_{j=1}^{t} y_{f 1}^{j}-1}\left(1-\theta_{f}\right)^{v_{2}+\sum_{j=1}^{t} y_{j / 2}^{j}-1}\right]}{\left(\tau_{f}+\theta_{f} \sum_{j=1}^{t} \gamma_{f 1}^{j}+\left(1-\theta_{f}\right) \sum_{j=1}^{t} \gamma_{f 2}^{j}\right)^{2 \tau_{f}+\sum_{i=1}^{j} \sum_{j=1}^{t} y_{j}^{j}+1}} d \theta_{f} .
\end{gathered}
$$


Calculating the integral, we obtain:

$$
\begin{aligned}
& E\left(\frac{\theta_{f i}}{\tau_{f}+\theta_{f} \sum_{j=1}^{t} \gamma_{f 1}^{j}+\left(1-\theta_{f}\right) \sum_{j=1}^{t} \gamma_{f 2}^{j}} \mid y_{f}, X_{f}\right)= \\
& \left(v_{i}+\sum_{j=1}^{t} y_{f i}^{j}\right)_{2} F_{1}\left(I+v_{i}+\sum_{j=1}^{t} y_{f 1}^{j} ; 1+2 \tau_{f}+\sum_{i=1}^{2} \sum_{j=1}^{t} y_{f i}^{j} ; 1+\sum_{i=1}^{2} v_{i}+\sum_{i=1}^{2} \sum_{j=1}^{t} y_{f i}^{j} ; \frac{\sum_{j=1}^{t} \gamma_{f 2}^{j}-\sum_{j=1}^{t} \gamma_{f 1}^{j}}{\tau_{f}+\sum_{j=1}^{t} \gamma_{f 2}^{j}}\right)(16) \\
& \left(\tau_{f}+\sum_{j=1}^{t} \gamma_{f 2}^{j}\right)\left(\sum_{j=1}^{t} v_{i}+\sum_{i=1}^{2} \sum_{j=1}^{t} y_{f i}^{j}\right){ }_{2} F_{1}\left(v_{i}+\sum_{j=1}^{t} y_{f 1}^{j} ; 2 \tau_{f}+\sum_{i=1}^{2} \sum_{j=1}^{t} y_{f i}^{j} ; \sum_{i=1}^{2} v_{i}+\sum_{i=1}^{2} \sum_{j=1}^{t} y_{f i}^{j} ; \frac{\sum_{j=1}^{t} \gamma_{f 2}^{j}-\sum_{j=1}^{j} \gamma_{f 1}^{j}}{\tau_{f}+\sum_{j=1}^{t} \gamma_{f 2}^{j}}\right)
\end{aligned}
$$

with $\theta_{f i}=\theta_{f}$ if $i=1$ and $1-\theta_{f}$ if $i=2$ and the indicative function $I=1$ if $i=1$ and $I=0$ if $i=2$.

Thus, the optimal estimator of vehicle $i$ is equal to:

$$
\begin{aligned}
\gamma_{f i}^{t+1}\left(\frac{E\left(\theta_{f i} \alpha_{f} \mid y_{f}, X_{f}\right)}{E\left(\alpha_{f}\right) E\left(\theta_{f i}\right)}\right) & = \\
& \gamma_{f i}^{t+1} \frac{v_{1}+v_{2}}{2 v_{i}}\left(2 \tau_{f}+\sum_{i=1}^{2} \sum_{j=1}^{t} y_{f i}^{j}\right) E\left(\frac{\theta_{f i}}{\tau_{f}+\theta_{f} \sum_{j=1}^{t} \gamma_{f i}^{j}+\left(1-\theta_{f}\right) \sum_{j=1}^{t} \gamma_{f 2}^{j}} \mid y_{f}, X_{f}\right),
\end{aligned}
$$

where the value of $E(\cdot)$ is given in (16).

We note that for each vehicle $i$, the optimal estimator for accidents at period $t+1$ is a function of the following factors: the parameters observable when the insurance policy is being renewed at period $t+1$; the accidents accumulated by vehicle $i$ over the preceding $t$ periods; the total accidents of the fleet over the same periods; the observable characteristics of the two vehicles over the preceding $t$ periods; and the gamma and Dirichlet parameters. To sum up, the term

$$
\frac{v_{1}+v_{2}}{2 v_{i}}\left(2 \tau_{f}+\sum_{i=1}^{2} \sum_{j=1}^{t} y_{f i}^{j}\right)
$$

measures the fleet contribution, while the term $E(\cdot)$ measures the truck contribution to the fleet. 
We shall apply this formula to our data in Section 4. But let's now see how it is possible to generalize this insurance rating formula to a fleet of $I_{f}$ vehicles.

\subsubsection{Carrier with more than 2 vehicles}

This section is divided into three subsections corresponding to the three approximation hypotheses for the multiple integral discussed in section 2.1.3.

- All the $\gamma_{f i}^{j}$ for the $I_{f}$ vehicles are identical

In this situation, the conditional accident probability for the fleet is given by:

$$
\begin{aligned}
P\left(y_{f} \mid \theta_{f 1}, \cdots, \theta_{f I_{f}-1}, \alpha_{f}, X_{f}\right) & =\prod_{i=1}^{I_{f}} \prod_{j=1}^{t} \frac{\left(y_{f i}^{j} \theta_{f i} \alpha_{f}\right)^{y_{f i}^{j}} e^{-\gamma_{f}^{j} \theta_{f i} \alpha_{f}}}{\Gamma\left(y_{f i}^{j}+1\right)} \\
& =\prod_{j=1}^{t} \prod_{i=1}^{I_{f}} \frac{\left(y_{f i}^{j}\right)^{y_{f i}^{j}}}{\Gamma\left(y_{f i}^{j}+1\right)} \prod_{i=1}^{I_{f}}\left(\theta_{f i}\right)^{\sum_{j=1}^{t} y_{f i}^{j}}\left(\alpha_{f}\right)^{\sum_{i=1}^{I_{f}} \sum_{j=1}^{t} \sum_{f}^{j}} e^{-\alpha_{f} \sum_{j=1}^{t} \gamma_{f}^{j}} .
\end{aligned}
$$

The optimal estimator $\hat{\lambda}_{f i}^{t+1}$ is thus equal to:

$$
\frac{\gamma_{f i}^{t+1} E\left(\theta_{f i} \alpha_{f} \mid y_{f}, X_{f}\right)}{E\left(\theta_{f i} \alpha_{f}\right)}=\gamma_{f i}^{t+1} \frac{\sum_{i=1}^{I_{f}} v_{i}}{I_{f} v_{i}}\left(\frac{v_{i}+\sum_{j=1}^{t} y_{f i}^{j}}{\tau_{f}+\sum_{j=1}^{t} \gamma_{f i}^{j}}\right)\left(\frac{I_{f} \tau_{f}+\sum_{i=1}^{I_{f}} \sum_{j=1}^{t} y_{f i}^{j}}{\sum_{i=1}^{I_{f}} v_{i}+\sum_{i=1}^{I_{f}} \sum_{j=1}^{t} y_{f i}^{j}}\right) .
$$

This formula compares rather well with the one presented in equation (13) for a carrier with a single vehicle. Here, as all the vehicles are identical in terms of the observable variables, differentiation of the two formulas will be principally the work of the experience variables. On the one hand, all the accidents of the fleet come into play and, on the other hand, the weight of past accidents takes into account the parameters of the Dirichlet distribution, on an individual basis $v_{i}$ for each vehicle and on an aggregated basis $\sum_{i=1}^{I_{f}} v_{i}$ for all the vehicles.

- Divide the vehicles into 2 groups

If we now have different vehicles, we can form groups with homogeneous characteristics or risks to obtain an explicit formula. In fact, insurers form more or less homogeneous risk classes by using different classification variables such as the type of car, the territory... Past experience serves to pinpoint the 
differences which are not observable a priori. If we limit ourselves to two groups, the conditional accident probability for the fleet is given by:

$$
\begin{aligned}
& P\left(y_{f} \mid \theta_{f 1}, \cdots, \theta_{f I_{f}-1}, \alpha_{f}, X_{f}\right)=\prod_{i=1}^{I_{f}} \prod_{j=1}^{t} \frac{\left(\gamma_{f i}^{j} \theta_{f i} \alpha_{f}\right)^{y_{f i}^{j}} e^{-\gamma_{f i}^{j} \theta_{j} \alpha_{f}}}{\Gamma\left(y_{f i}^{j}+1\right)}
\end{aligned}
$$

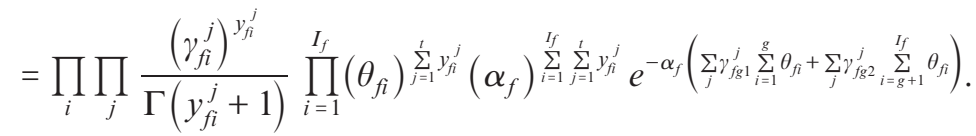

with

$$
\gamma_{f g 1}^{j}=\left(\frac{\sum_{i=1}^{g} \gamma_{f i}^{j}}{g}\right) \text { and } \gamma_{f g 2}^{j}=\left(\frac{\sum_{i=g+1}^{I_{f}} \gamma_{f i}^{j}}{I_{f}-g}\right)
$$

for the two groups respectively.

The optimal estimator $\hat{\lambda}_{f i}^{t+1}$ is thus equal to:

$$
\begin{aligned}
& \left.\gamma_{f i}^{t+1}\left(\frac{E\left(\theta_{f i} \alpha_{f} \mid y_{f}, X_{f}\right)}{E\left(\alpha_{f}\right) E\left(\theta_{f i}\right)}\right)=\gamma_{f i}^{t+1} \frac{\sum_{i=-1}^{I_{f}} v_{i}}{I_{f} v_{i}}\left(I_{f} \tau_{f}+\sum_{i=1}^{I_{f}} \sum_{j=1}^{t} y_{f i}^{j}+\right) E\left(\frac{\theta_{f i}}{\tau_{f}+\gamma_{f g 1}^{j} \sum_{m=1}^{g} \theta_{f m}+\gamma_{f g 2}^{j} \sum_{m=g+1}^{t} \theta_{f m}}\right) y_{f}, X_{f}\right) \\
& =\gamma_{f i}^{t+1} \frac{\sum_{i=1}^{I_{f}} v_{i}}{I_{f} v_{i}}\left(I_{f} \tau_{f}+\sum_{i=1}^{I_{f}} \sum_{j=1}^{t} y_{f i}^{j}\right) \times \\
& \left(v_{i}+\sum_{j=1}^{t} y_{f i}^{j}\right) 2 F_{1}\left(I+\sum_{i=1}^{g} v_{i}+\sum_{i=1}^{g} \sum_{j=1}^{t} y_{f i}^{j} ; 1+I_{f} \tau_{f}+\sum_{i=1}^{I_{f}} \sum_{j=1}^{t} y_{f i}^{j} ; 1+\sum_{i=1}^{I_{f}} v_{i}+\sum_{i=1}^{I_{f}} \sum_{j=1}^{t} y_{f i}^{j} ; \frac{\left.\sum_{j=1}^{t} \gamma_{f g 2}^{j}-\sum_{j=1}^{t} \gamma_{f g 1}^{j}\right)}{\left.\tau_{f}+\sum_{j=1}^{t} \gamma_{f g 2}^{j}\right)}\right. \\
& \left(\tau_{f}+\sum_{j=1}^{t} \gamma_{f g 2}^{j}\right)\left(\sum_{i=1}^{I_{f}} v_{i}+\sum_{i=1}^{I_{f}} \sum_{j=1}^{t} y_{f i}^{j}\right) F_{2} F_{1}\left(\sum_{i=1}^{g} v_{i}+\sum_{i=1}^{g} \sum_{j=1}^{t} y_{f i}^{j} ; I_{f} \tau_{f}+\sum_{i=1}^{t} \sum_{j=1}^{t} y_{f i}^{j} ; \sum_{i=1}^{I_{f}} v_{i}+\sum_{i=1}^{I_{f}} \sum_{j=1}^{t} y_{f i}^{j} ; \frac{j}{\tau_{f}+\sum_{j=1}^{t} \gamma_{f g 2}^{j}}\right)
\end{aligned}
$$

where the indicative variable:

$$
I=\left\{\begin{array}{l}
1 \text { if the truck belongs to group } 1 \\
0 \text { if the truck belongs to group } 2 .
\end{array}\right.
$$


This formula is very difficult to generalize to more than two groups. If the fleet has several more or less homogeneous groups of vehicles, it may be more advantageous to rely on a Monte Carlo simulation approach.

- Monte Carlo method

In the general case, we must evaluate the following density function:

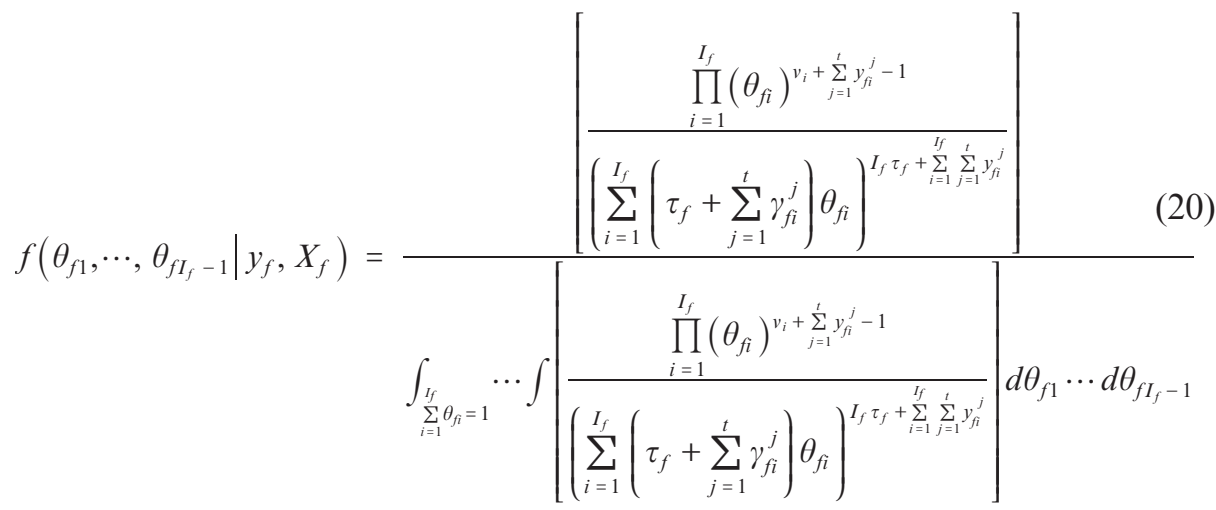

We can estimate the multiple integral at the denominator of equation (20) with the Monte Carlo method by using the importance (weighting) function (Lange, 1999) as shown in Section 2.1.3.

By taking:

$$
h(\underset{\sim}{\theta})=\frac{\Gamma\left(\sum_{i=1}^{I_{f}} v_{i}+\sum_{i=1}^{I_{f}} \sum_{j=1}^{t} y_{f i}^{j}\right)}{\prod_{i=1}^{I_{f}} \Gamma\left(v_{i}+\sum_{i=1}^{t} y_{f i}^{j}\right)} \prod_{i=1}^{I_{f}}\left(\theta_{f i}\right)^{v_{i}+\sum_{j=1}^{t} y_{f}^{j}-1},
$$

the optimal estimator $\hat{\lambda}_{f i}^{t+1}$ can be approximated by:

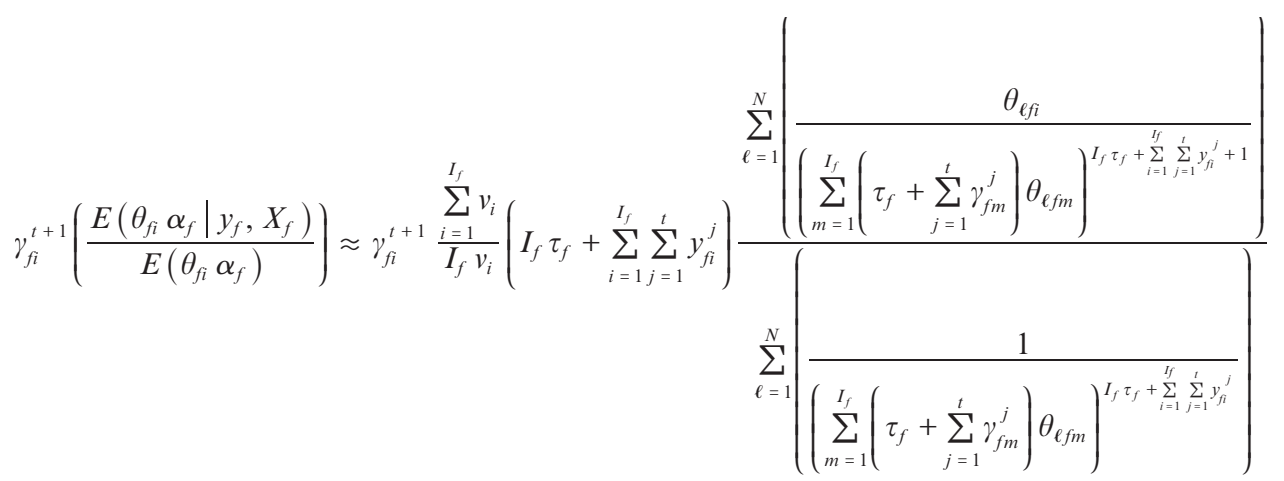


with

$$
\theta_{\ell f i}=\frac{a_{\ell f i}}{\sum_{i=1}^{I_{f}} a_{\ell f i}},
$$
where the $a_{\ell f i}$ are values from the gamma density with parameters $\left(v_{i}+\sum_{j=1}^{t} y_{f i}^{j}, 1\right)$ for
$i=1, \ldots, I_{f}$ and $\ell=1, \ldots, N$.

\section{Application of the Bonus malus System}

In this section, we propose premium tables over several years, representing extensions of those proposed in the literature on automobile insurance for individual vehicles. Given that we did not model the conditional distribution for the cost of claims, we suppose that the average cost of claims is $\$ 10,000$, seemingly a reasonable value for accidents involving trucks in North America (Dionne, Laberge-Nadeau et al., 1999).

\subsection{Fleet of 2 trucks}

Table 3A presents an example of premiums calculated for a truck belonging to a fleet of two trucks. The first line of the table (Fleet accidents) gives the sum of the accidents for the fleet over $t$ years. The maximum indicated is 2 accidents but it could be higher. The second line (Truck accidents) gives the sum of accidents for the truck in question. For example, in the third column where the fleet accumulates two accidents, the truck concerned may have had 0,1 or 2 accidents. Thus each corresponding scenario of premiums depends on the truck's and the fleet's own experience. In this section, we apply the formula in (19) along with the result of Table 1 showing that $v_{1}=v_{2}=v$. This formula measures the product of the BMF with the a priori value $\gamma_{f i}^{t+1}$.

The estimated values of the parameters are equal to $\hat{\tau}_{f}^{-1}=1.5615$ and $\hat{v}=2.2056$ (Table 1). Let's take the column "No accident" in Table 3A for the fleet and the trucks. We note that the premium for the trucks decreases over time. The following column gives the variations in the premiums if the fleet does have an accident and depending on whether or not the truck has an accident. We note that the premium for the truck increases in comparison to the first column even if the truck did not have an accident, for it is penalized by the fleet effect. But the increase is less than the one corresponding to the case where it did incur an accident. For example, if the fleet has one accident with truck 2, the BMF 


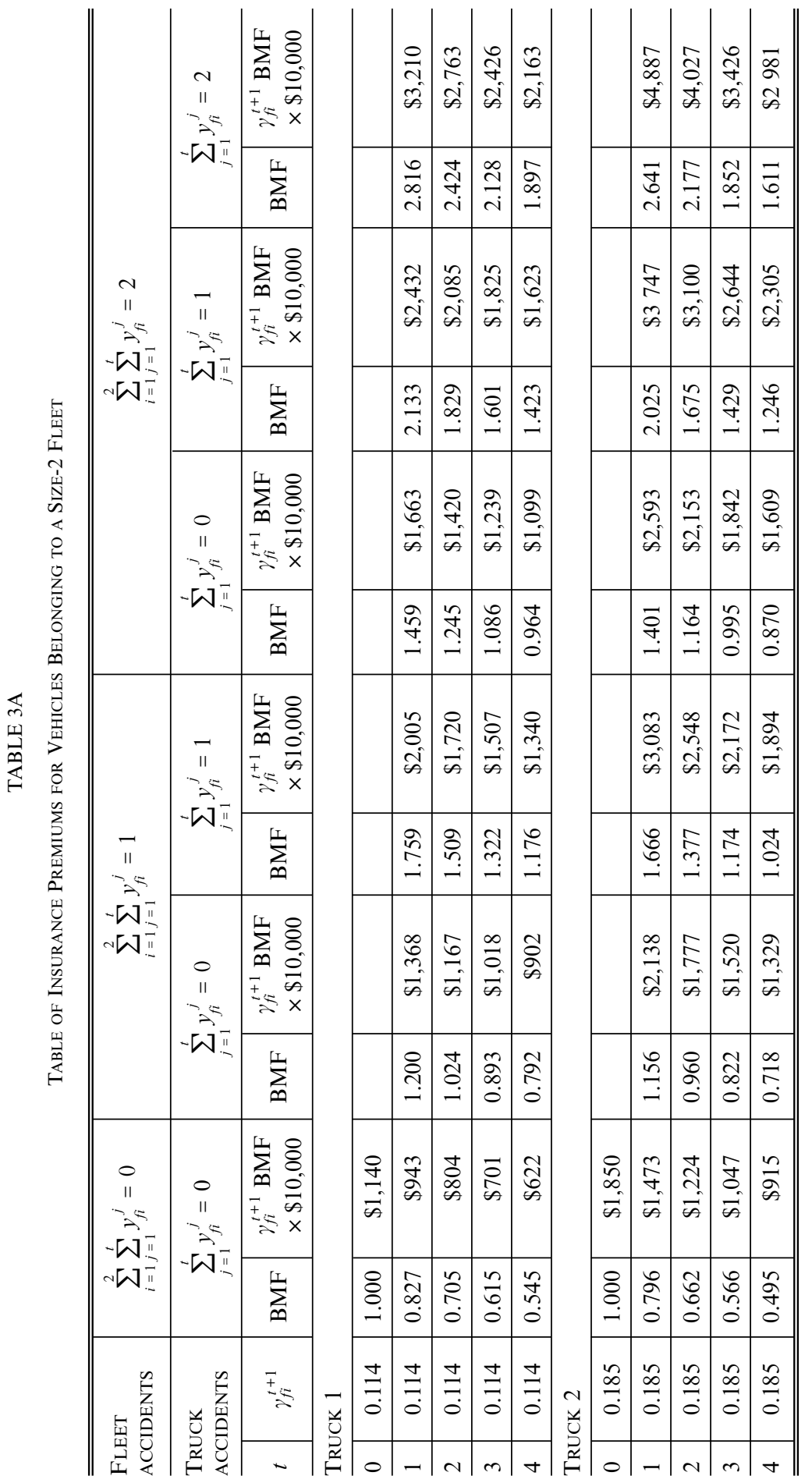


of the first truck will go from 0.827 to 1.2 , while that of the second truck will go from 0.796 to 1.666 .

A direct link can be made between the BMF and the predictive probabilities computed in Section 2.2.3. In fact, the BMF can be written as

$$
\mathrm{BMF}=\frac{E\left[Y_{f i}^{t+1} \mid Y_{f 1}^{t} Y_{f 2}^{t}\right]}{E\left[Y_{i}^{t}\right]} .
$$

Using the numbers in Table 2A for truck 1 with no accident at $t$ for the fleet, we obtain:

$$
\mathrm{BMF}=\frac{0.0942558}{0.114}=0.827,
$$

which is exactly the corresponding BMF in Table 3A.

Table 3B compares the results with the semiparametric method for $t=1$. The two semiparametric models come from Dionne et al. (2001). In the model of Section 3.3 in Dionne et al. (2001), the credibility related to the history of a vehicle is computed at the fleet level, while, in the model of Section 3.5, it is computed for each vehicle. We observe that the variations of the BMF differ. The variations of the BMF conditional on past experience are wider in the parametric model than in the semiparametric ones. Since the residuals are smaller in absolute value (Median and IQR in Table 2) in the parametric model than in the Poisson model (44 estimated parameters instead of 36), the dispersion of the premiums adjustments increases or introduces more incentives for road safety. We make other comparisons between the two approaches with data from a larger fleet in the last section of the paper.

TABLE 3B

COMPARISON WITH SEMIPARAMETRIC MODEL

\begin{tabular}{l|c|c|c|c|c|c}
\hline \hline & \multirow{2}{*}{ Bonus malus coefficient } & \multicolumn{2}{|c|}{$\sum_{i=1}^{2} y_{i}=0$} & \multicolumn{2}{|c}{$\sum_{i=1}^{2} y_{i}$} & \multicolumn{3}{|c}{$\sum_{i=1}^{2} y_{i}=2$} \\
\cline { 3 - 7 } & & $y_{i}=0$ & $y_{i}=1$ & $y_{i}=0$ & $y_{i}=1$ & $y_{i}=2$ \\
\hline See Tableau 2 (truck 1) & 0.827 & 1.200 & 1.759 & 1.459 & 2.133 & 2.816 \\
\hline $\begin{array}{l}\text { Section 3.5 } \\
\text { Dionne et al., 2001 }\end{array}$ & 0.876 & 1.247 & 1.594 & 1.619 & 1.966 & 2.312 \\
\hline $\begin{array}{l}\text { Section 3.3 } \\
\text { Dionne et al., 2001 }\end{array}$ & 0.876 & 1.421 & 1.421 & 1.966 & 1.966 & 1.966 \\
\hline \hline
\end{tabular}




\subsection{Fleet of several trucks}

\subsubsection{All vehicles in fleet have the same risk characteristics}

In this situation, the insurance premium estimated for truck $i$ belonging to carrier $f$ is given by (18) with $\sum_{i=1}^{I_{f}} v_{i}=I_{f} \hat{v}$.

Table 4 presents this example for a fleet of 10 identical trucks with $\hat{v}=2.2056$ and $\hat{\tau}_{f}^{-1}=6.4867$ (see Table 1). Suppose that the carrier accumulates 2 accidents over the next period, with 6 trucks incurring no accident nor speeding violation; 2 trucks incurring no accident but one speeding violation; 1 truck incurring an accident but no speeding violation; and 1 truck incurring an accident as well as a speeding violation. Still supposing that the average cost of claims is $\$ 10,000$, the a priori insurance premium for a vehicle when no account is taken of past experience is established at $\$ 1,850(0.185 \times 1 \times \$ 10,000)$. Since all the vehicles of the fleet are identical in terms of observable risk, they all have the same $\gamma_{f i}^{t}=0.185$ and a BMF equal to 1 at the start of the insurance contract. The total premium for the fleet is established at $\$ 18,500(10 \times \$ 1,850)$. In the following period $(t+1)$, the insurance premiums for each of the records of the vehicles in the fleet are given in Table 4.

TABLE 4

Table of Insurance Premiums for Vehicles Belonging to a Size-10 Fleet When the FleEt Accumulated 2 Accidents and 2 Violations

\begin{tabular}{c|c|c|c|c|c|c|c}
\hline \hline$\gamma_{f i}^{t}$ & $\begin{array}{c}\text { Accumulation } \\
\text { of accidents }\end{array}$ & $\begin{array}{c}\text { Speeding } \\
\text { violation }\end{array}$ & $\gamma_{f i}^{t+1}$ & BMF & $\begin{array}{c}\gamma_{f i}^{t+1} \text { BMF } \\
\times \$ 10,000\end{array}$ & $\begin{array}{c}\text { Number of } \\
\text { trucks }\end{array}$ & \\
\hline 0.185 & 0 & 0 & 0.185 & 0.957 & $\$ 1,770$ & 6 & $\$ 10,620$ \\
\hline 0.185 & 0 & 1 & 0.324 & 0.957 & $\$ 3,101$ & 2 & $\$ 6,202$ \\
\hline 0.185 & 1 & 0 & 0.185 & 1.391 & $\$ 2,573$ & 1 & $\$ 2,573$ \\
\hline 0.185 & 1 & 1 & 0.324 & 1.391 & $\$ 4,507$ & 1 & $\$ 4,507$ \\
\hline Total & 2 & 2 & & & & 10 & $\$ 23,902$ \\
\hline \hline
\end{tabular}

We observe that the BMF is higher for vehicles having an accident than for those which did not. We note that accidents affect the BMF of all the vehicles (fleet effect). We also notice that the a priori risk measurement $\gamma_{f i}^{t+1}$ increases significantly for vehicles which have accumulated a speeding violation. If none of the 10 vehicles in the fleet had been involved in an accident nor had been charged with speeding, the total premium would have decreased from $\$ 18,500$ to $\$ 8,440(10 \times \$ 844)$, for the $\mathrm{BMF}$ would be equal to 0.456 and the individual truck premium to $\$ 844(0.185 \times 0.456 \times \$ 10,000=\$ 844)$. However, in our example, the total premium goes from $\$ 18,500$ to $\$ 23,902$ based on the accumulated experience of the 10 vehicles. 
Now, if the carrier has accumulated 3 past accidents and has 9 trucks with no accident and no speeding violation and 1 truck with 3 accidents but no speeding violation, the total premium is $\$ 24,776$. The insurance premiums of the fleet for each of the experiences are given in Table 5.

TABLE 5

Table of Insurance Premiums for Vehicles Belonging to a Size-10 Fleet When the Fleet has ACCumulated 3 ACCidents

\begin{tabular}{c|c|c|c|c|c|c}
\hline \hline$\gamma_{f i}^{t}$ & $\begin{array}{c}\text { Accumulation } \\
\text { of accidents }\end{array}$ & $\gamma_{f i}^{t+1}$ & BMF & $\begin{array}{c}\gamma_{f i}^{t+1} \mathrm{BMF} \\
\times \$ 10,000\end{array}$ & $\begin{array}{c}\text { Number of } \\
\text { trucks }\end{array}$ & \\
\hline 0.185 & 0 & 0.185 & 1.179 & $\$ 2,181$ & 9 & $\$ 19,629$ \\
0.185 & 3 & 0.185 & 2.782 & $\$ 5,147$ & 1 & $\$ 5,147$ \\
Total & 3 & & & & 10 & $\$ 24,776$ \\
\hline \hline
\end{tabular}

We note that the premium for a vehicle with no accident nor speeding violation is $\$ 2,181$ when it belongs to a fleet having accumulated 3 accidents and drops to $\$ 1,770$ if it belongs to a fleet having accumulated 2 accidents, while retaining the same characteristics (Table 4). This result is explained by the fact that the BMFs of all the vehicles are affected by the fleet's accumulation of accidents. We also note that accumulating 3 accidents increases the insurance premium more $(\$ 5,147)$ than accumulating one accident and one speeding violation $(\$ 4,507)$.

\subsubsection{Dividing the vehicles into 2 groups}

In this situation, the estimated insurance premium of a truck $i$ belonging to a carrier $f$ is given by (19) with $\sum_{i=1}^{I_{f}} v_{i}=I_{f} \hat{v}$.

Suppose that the accidents accumulated by the carrier over the next period is 0 , with 4 trucks belonging to group 1 (a priori expected number of accidents below or equal to 0.14345 ) and 6 trucks belonging to group 2 (a priori expected number of accidents above 0.14345). By supposing that the average cost of claims is $\$ 10,000$, the insurance premiums for the history of each of the fleet's vehicles in the following period are given in Table 6.

Now, if the fleet has accumulated 1 accident and if the vehicle involved in the accident belongs to group 2, the insurance premiums for the fleet's vehicles are given in Table 7 .

In contrast, if the vehicle involved in the accident belongs to group 1, we obtain the values shown in Table 8 .

Table 9 sums up all the cases (numbers not in parentheses) with the hypergeometric method, as well those with the Monte Carlo method (number in parentheses). 
TABLE 6

Insurance Premiums for Vehicles Belonging to a 10-Truck Fleet When the Fleet has not Accumulated a Single Accident

\begin{tabular}{c|c|c|c|c|c|c|c}
\hline \hline Group & $\hat{\gamma}_{f g i}^{t}$ & $\begin{array}{c}\text { Accumulation } \\
\text { of accidents }\end{array}$ & $\gamma_{f i}^{t+1}$ & BMF & $\begin{array}{c}\gamma_{f i}^{t+1} \mathrm{BMF} \\
\times \$ 10,000\end{array}$ & $\begin{array}{c}\text { Number of } \\
\text { trucks }\end{array}$ & \\
\hline 1 & 0.1305 & 0 & 0.1305 & 0.455 & $\$ 594$ & 4 & $\$ 2,376$ \\
2 & 0.2331 & 0 & 0.2331 & 0.440 & $\$ 1,026$ & 6 & $\$ 6,156$ \\
Total & 0 & & & & 10 & $\$ 8,532$ \\
\hline \hline
\end{tabular}

TABLE 7

Table of Insurance Premiums for Vehicles Belonging to a 10-Truck Fleet When the Fleet has ACCumulated 1 ACCident (IN GROUP 2)

\begin{tabular}{c|c|c|c|c|c|c|c}
\hline \hline Group & $\hat{\gamma}_{f g i}^{t}$ & $\begin{array}{c}\text { Accumulation } \\
\text { of accidents }\end{array}$ & $\gamma_{f i}^{t+1}$ & BMF & $\begin{array}{c}\gamma_{f i}^{t+1} \mathrm{BMF} \\
\times \$ 10,000\end{array}$ & $\begin{array}{c}\text { Number of } \\
\text { trucks }\end{array}$ & \\
\hline 1 & 0.1305 & 0 & 0.1305 & 0.720 & $\$ 940$ & 4 & $\$ 3,760$ \\
2 & 0.2331 & 0 & 0.2331 & 0.689 & $\$ 1,606$ & 5 & $\$ 8,030$ \\
2 & 0.2331 & 1 & 0.2331 & 1.001 & $\$ 2,333$ & 1 & $\$ 2,333$ \\
Total & 1 & & & & 10 & $\$ 14,123$ \\
\hline \hline
\end{tabular}

TABLE 8

Table of Insurance Premiums for Vehicles Belonging to a 10-Truck Fleet When the FleEt has ACCumulated 1 ACCident (IN Group 1)

\begin{tabular}{c|c|c|c|c|c|c|c}
\hline \hline Group & $\hat{\gamma}_{f g i}^{t}$ & $\begin{array}{c}\text { Accumulation } \\
\text { of accidents }\end{array}$ & $\gamma_{f i}^{t+1}$ & BMF & $\begin{array}{c}\gamma_{f i}^{t+1} \mathrm{BMF} \\
\times \$ 10,000\end{array}$ & $\begin{array}{c}\text { Number of } \\
\text { trucks }\end{array}$ & \\
\hline 1 & 0.1305 & 0 & 0.1305 & 0.728 & $\$ 950$ & 3 & $\$ 2,850$ \\
1 & 0.1305 & 1 & 0.1305 & 1.058 & $\$ 1,381$ & 1 & $\$ 1,381$ \\
2 & 0.2331 & 0 & 0.2331 & 0.697 & $\$ 1,625$ & 6 & $\$ 9,750$ \\
Total & 1 & & & & 10 & $\$ 13,981$ \\
\hline \hline
\end{tabular}

It should be noted that the Monte Carlo computations of premiums are identical to those with the hypergeometric approximation when we assume that all trucks are identical inside the two groups. They correspond to the numbers in parentheses in Table 9. One advantage of the Monte Carlo method is that we can use it to consider all trucks as different in a given fleet. We now present results for ten different trucks, using the Monte Carlo method to make a posteriori computations. We use the econometric results of Table 1 for a priori evaluations. The iterations are repeated $n=500,000$ times; this takes about 10 minutes for a scenario like the one presented in Table 10, whereas the hypergeometric approximations are instantaneous. 


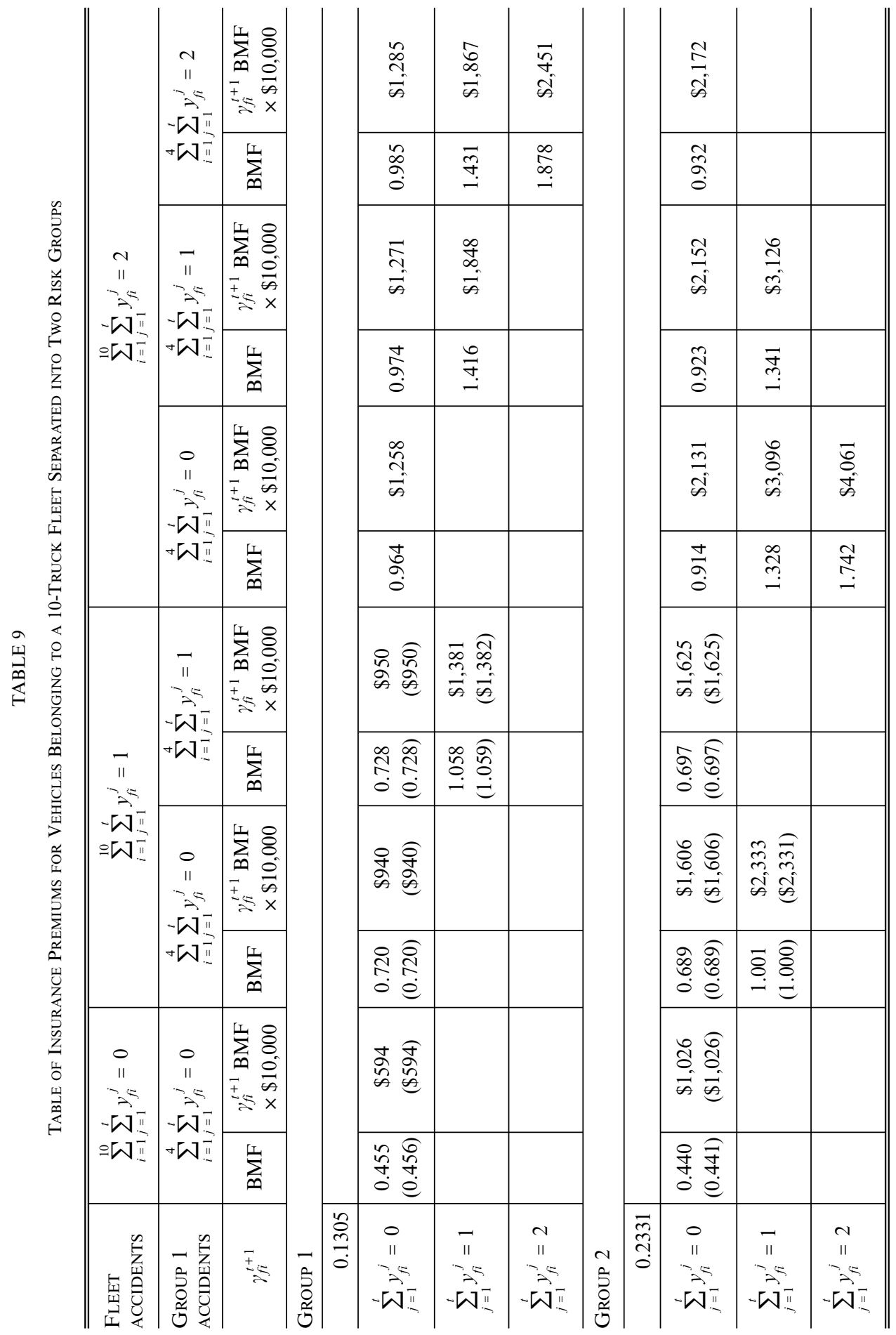




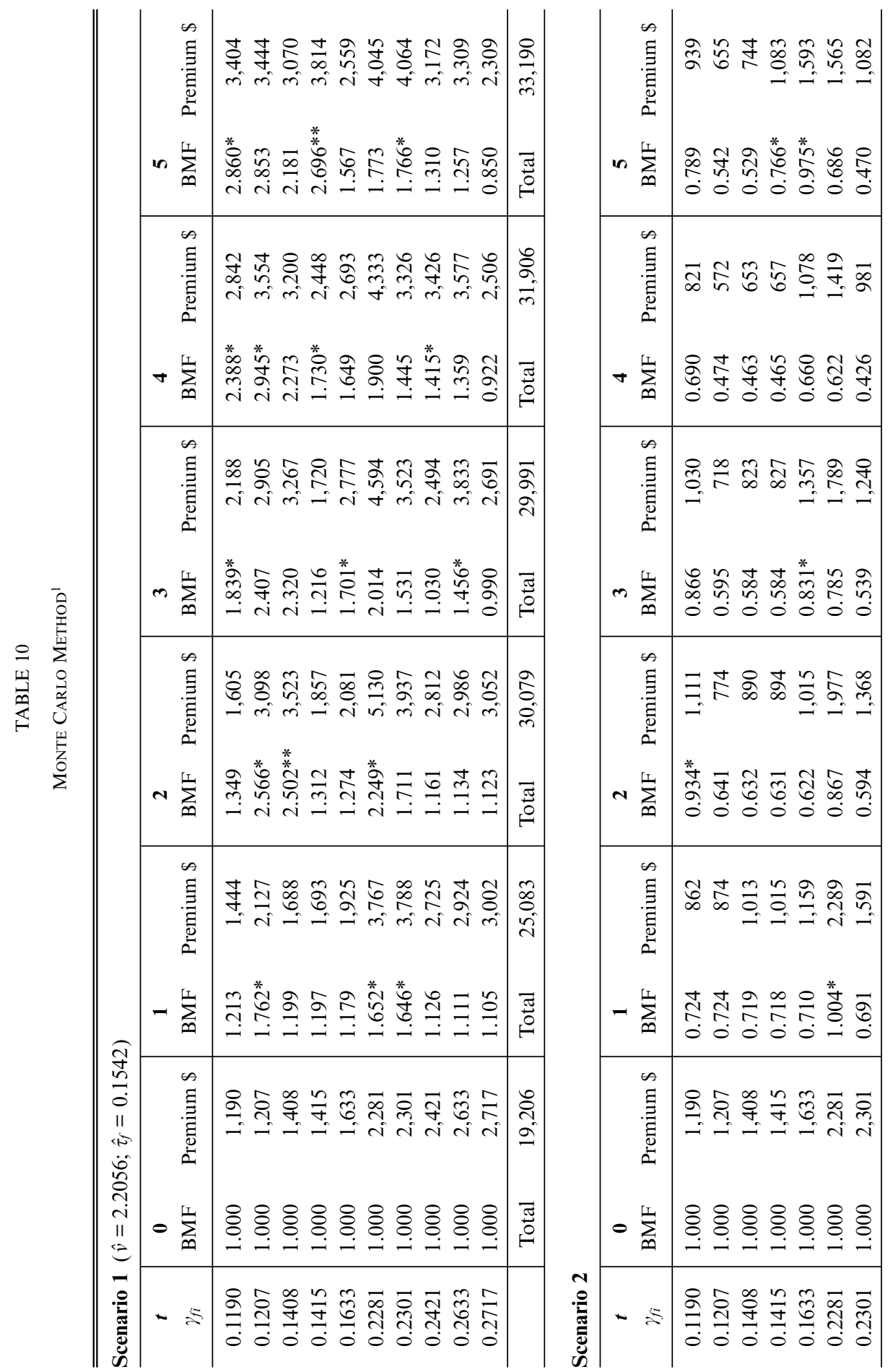




\begin{tabular}{|c|c|c|}
\hline 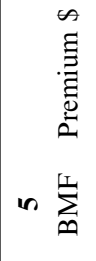 & 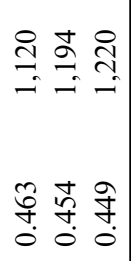 & $\begin{array}{l}\stackrel{\circ}{\Xi} \\
\stackrel{\Xi}{0}\end{array}$ \\
\hline 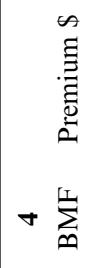 & 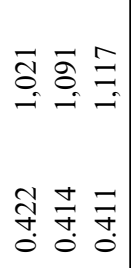 & $\frac{\pi}{0}$ \\
\hline 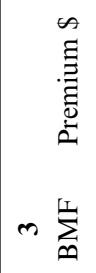 & 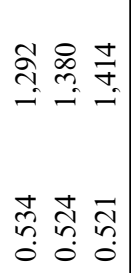 & $\frac{\pi}{0}$ \\
\hline 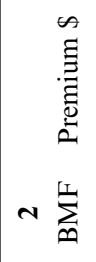 & 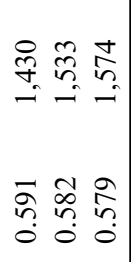 & 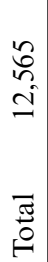 \\
\hline 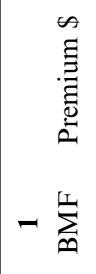 & 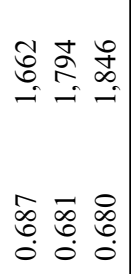 & 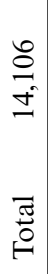 \\
\hline 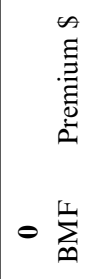 & 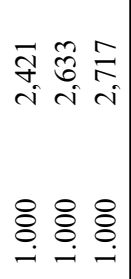 & 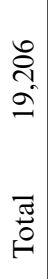 \\
\hline$-\Sigma$ & 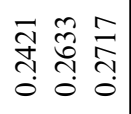 & \\
\hline
\end{tabular}

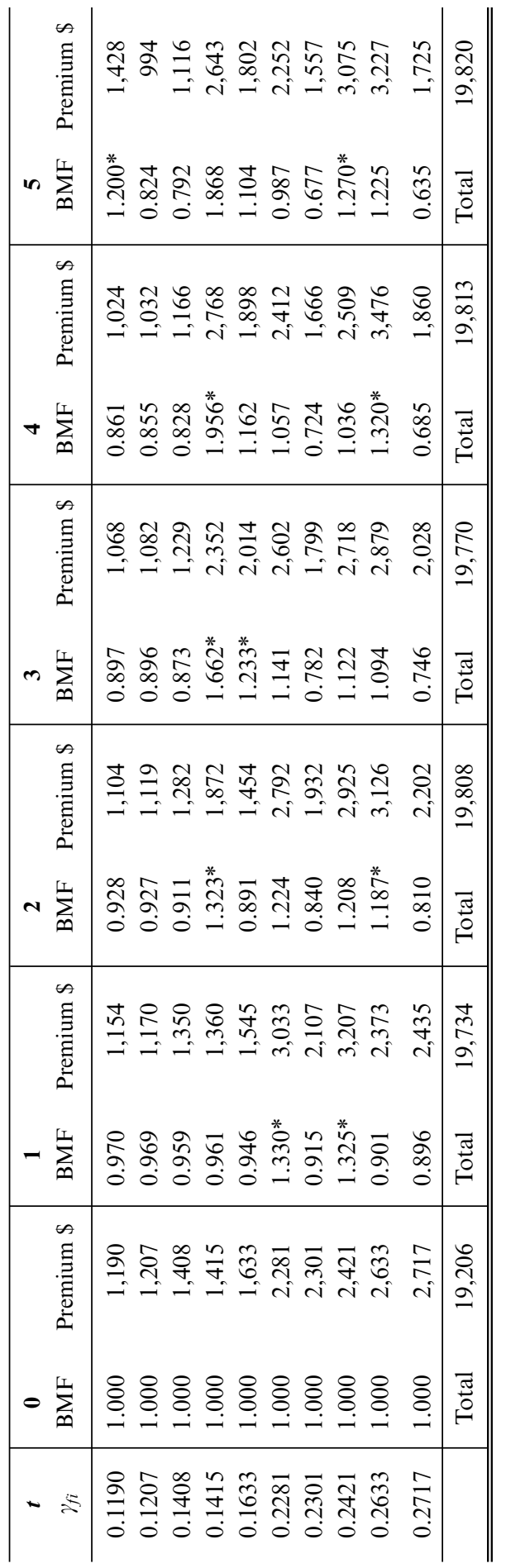

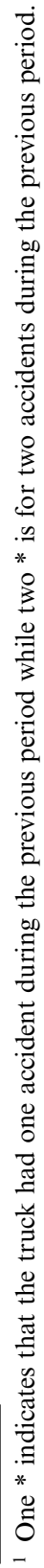




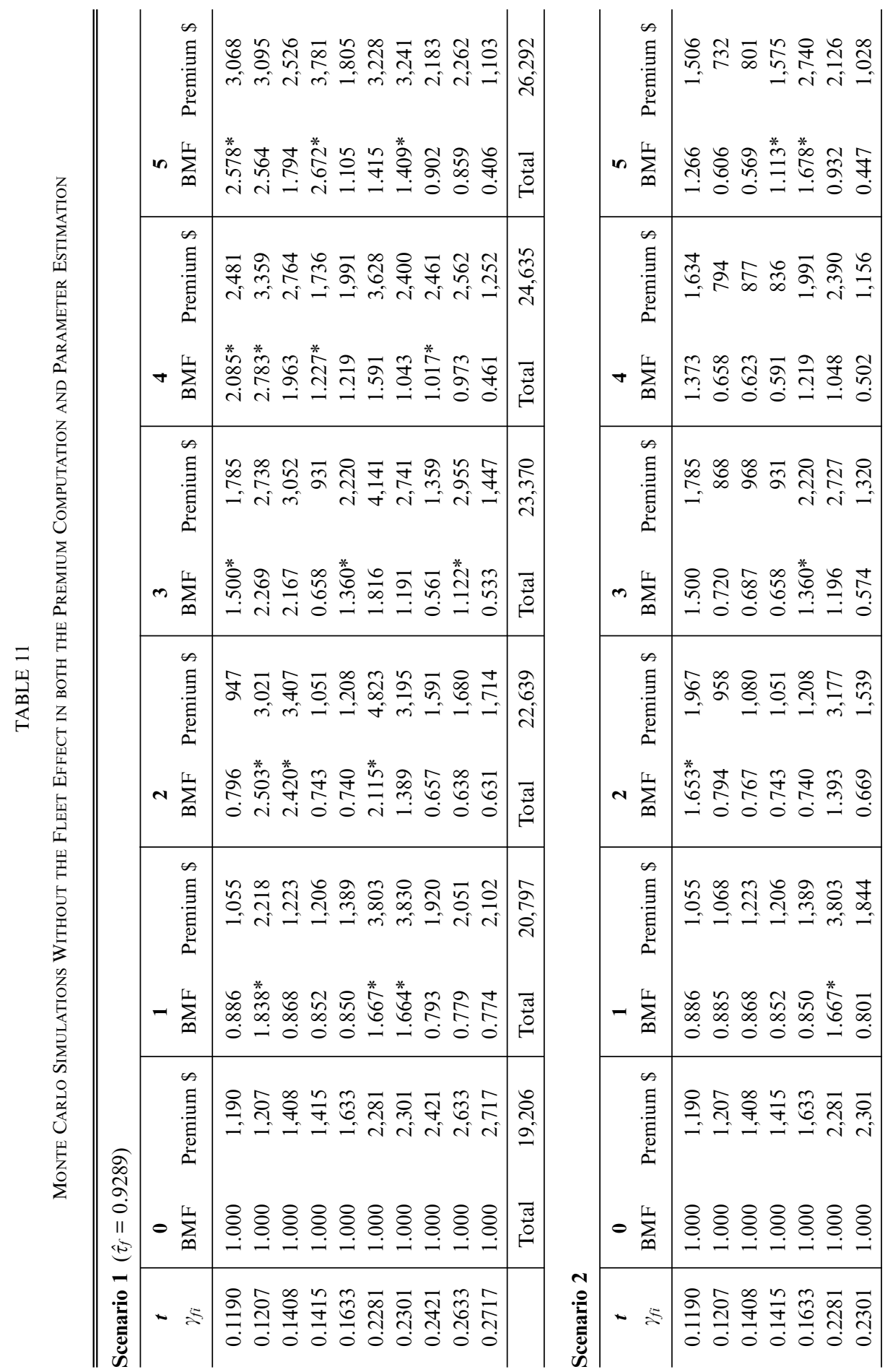




\begin{tabular}{|c|c|c|}
\hline 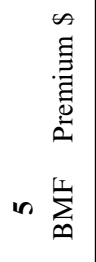 & 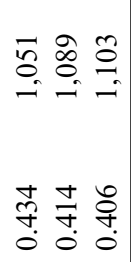 & $\begin{array}{l}\frac{\pi}{2} \\
2 \\
\frac{\pi}{0} \\
0\end{array}$ \\
\hline 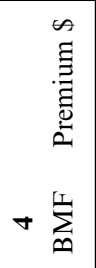 & 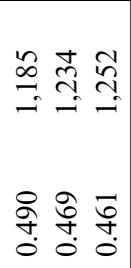 & 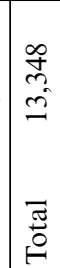 \\
\hline 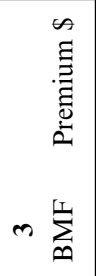 & 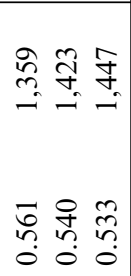 & $\begin{array}{l}0 \\
0 \\
0 \\
0 \\
0 \\
0\end{array}$ \\
\hline 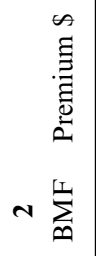 & 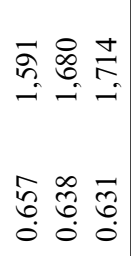 & $\begin{array}{l}0 \\
8 \\
2 \\
2 \\
3 \\
0 \\
0\end{array}$ \\
\hline 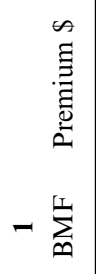 & 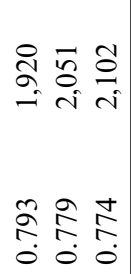 & 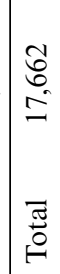 \\
\hline 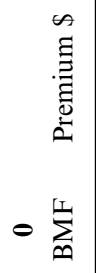 & \& \& \& & 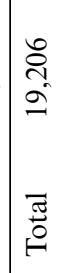 \\
\hline$-\Sigma$ & 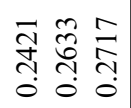 & \\
\hline
\end{tabular}

\begin{tabular}{|c|c|c|}
\hline 事 & 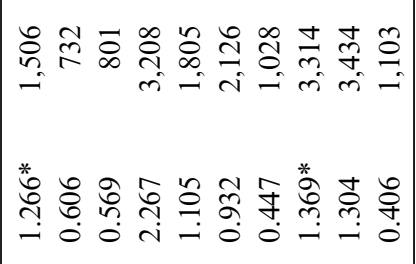 & $\begin{array}{l}\infty \\
2 \\
2 \\
2\end{array}$ \\
\hline 泀 & 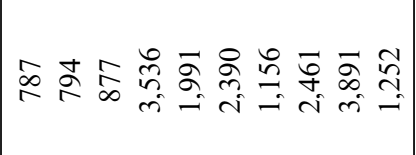 & $\frac{\tilde{m}}{2}$ \\
\hline$+\sum_{m}^{I}$ & 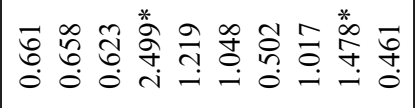 & 胥 \\
\hline 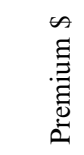 & 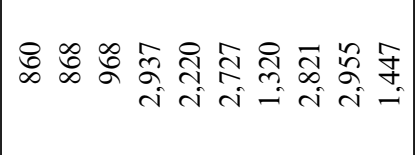 & $\stackrel{\overbrace{}}{\varrho}$ \\
\hline$m \sum_{\infty}^{L}$ & 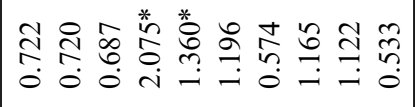 & : \\
\hline$\stackrel{\infty}{\Xi}$ & 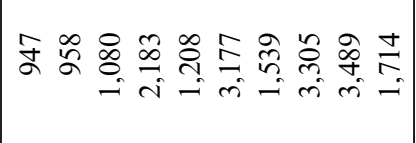 & $\begin{array}{l}8 \\
8 \\
0\end{array}$ \\
\hline$\sim \sum_{m}^{I}$ & 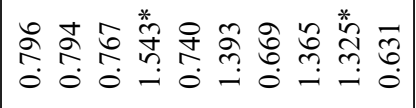 & 吾 \\
\hline 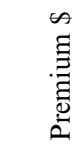 & 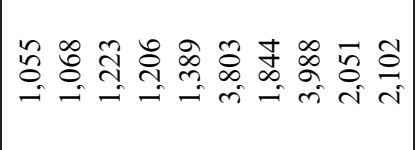 & $\frac{\grave{\triangleq}}{a}$ \\
\hline$-\sum_{\infty}^{1}$ & 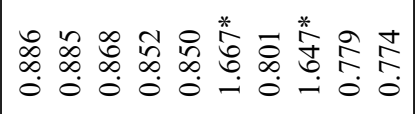 & ॠే \\
\hline$\stackrel{\infty}{\Xi}$ & 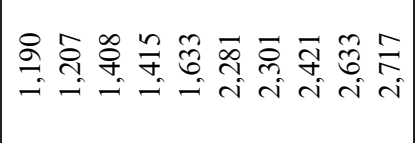 & $\begin{array}{l}\stackrel{\leftrightarrow}{1} \\
2\end{array}$ \\
\hline$\ominus \sum_{\infty}^{1}$ & 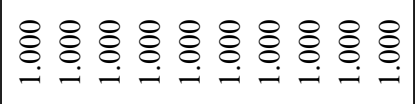 & 吾 \\
\hline - & 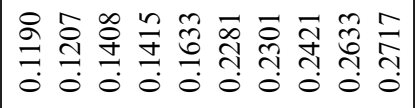 & \\
\hline
\end{tabular}


Table 10 presents the premium evolution over five years for three scenarios. The a priori expected number of accidents for these three scenarios is 0.192 . Scenario 1 is for a fleet that accumulates many accidents over time. In the first column, we observe the ten different a priori values. In the third column, we have the corresponding starting premiums for the three scenarios which amount to a total premium of $\$ 19,206$ for the fleet. Accumulating eighteen accidents over five years yields a total premium of $\$ 33,190$ for the next period. In scenario 2, the fleet accumulates only five accidents over the five years and the total premium drops to $\$ 11,196$. Finally, in scenario 3, the fleet has two

TABLE 12

Comparison of BMF and Premiums for FleEts of 10 Trucks Between Parametric and Semiparametric Models

Scenario 1 with $t=1$

\begin{tabular}{c|c|c|c|c|c}
\hline \hline & \multicolumn{2}{|c|}{ Equation 22 } & \multicolumn{2}{c|}{ Section 3.5 Dionne et al., 2001 } & \\
\hline$\gamma_{f i}^{t+1}$ & BMF & Premium (\$) & BMF & Premium (\$) & Number of Accident \\
\hline 0,1190 & 1,213 & 1444 & 1,220 & 1452 & 0 \\
0,1207 & 1,762 & 2127 & 1,432 & 1729 & 1 \\
0,1408 & 1,199 & 1688 & 1,216 & 1712 & 0 \\
0,1415 & 1,197 & 1693 & 1,216 & 1720 & 0 \\
0,1633 & 1,179 & 1925 & 1,211 & 1978 & 0 \\
0,2281 & 1,652 & 3767 & 1,406 & 3206 & 1 \\
0,2301 & 1,646 & 3788 & 1,405 & 3233 & 0 \\
0,2421 & 1,126 & 2725 & 1,195 & 2894 & 0 \\
0,2633 & 1,111 & 2924 & 1,191 & 3136 & 0 \\
0,2717 & 1,105 & 3002 & 1,190 & 3232 & 3 \\
\hline
\end{tabular}

Scenario 2 with $t=1$

\begin{tabular}{c|c|c|c|c|c}
\hline \hline & \multicolumn{2}{|c|}{ Equation 22 } & \multicolumn{2}{c}{ Section 3.5 Dionne et al., 2001 } & \\
\hline$\gamma_{f i}^{t+1}$ & BMF & Premium (\$) & BMF & Premium (\$) & Number of Accident \\
\hline 0,1190 & 0,724 & 862 & 0,767 & 913 & 0 \\
0,1207 & 0,724 & 874 & 0,767 & 926 & 0 \\
0,1408 & 0,719 & 1013 & 0,763 & 1074 & 0 \\
0,1415 & 0,718 & 1015 & 0,763 & 1079 & 0 \\
0,1633 & 0,710 & 1159 & 0,758 & 1238 & 0 \\
0,2281 & 1,004 & 2289 & 0,953 & 2173 & 1 \\
0,2301 & 0,691 & 1591 & 0,745 & 1714 & 0 \\
0,2421 & 0,687 & 1662 & 0,742 & 1797 & 0 \\
0,2633 & 0,681 & 1794 & 0,738 & 1944 & 0 \\
0,2717 & 0,680 & 1846 & 0,737 & 2001 & 1 \\
\hline & Total & 14106 & Total & 14858 & 0 \\
\hline \hline
\end{tabular}


Scenario 3 with $t=1$

\begin{tabular}{c|c|c|c|c|c}
\hline \hline & \multicolumn{2}{|c|}{ Equation 22 } & \multicolumn{2}{c|}{ Section 3.5 Dionne et al., 2001 } & \\
\hline$\gamma_{f i}^{t+1}$ & BMF & Premium (\$) & BMF & Premium (\$) & Number of Accident \\
\hline 0,1190 & 0,970 & 1154 & 0,988 & 1176 & 0 \\
0,1207 & 0,969 & 1170 & 0,988 & 1192 & 0 \\
0,1408 & 0,959 & 1350 & 0,984 & 1385 & 0 \\
0,1415 & 0,961 & 1360 & 0,983 & 1392 & 0 \\
0,1633 & 0,946 & 1545 & 0,979 & 1599 & 0 \\
0,2281 & 1,330 & 3033 & 1,174 & 2677 & 1 \\
0,2301 & 0,915 & 2107 & 0,966 & 2222 & 0 \\
0,2421 & 1,325 & 3207 & 1,170 & 2833 & 0 \\
0,2633 & 0,901 & 2373 & 0,959 & 2525 & 0 \\
0,2717 & 0,896 & 2435 & 0,958 & 2602 & 2 \\
\hline & Total & 19734 & Total & 19603 & 0 \\
\hline \hline
\end{tabular}

accidents each year (its average), resulting in an almost constant premium over time.

In Figure 1, we graphically represent the three scenarios with solid lines. The dotted lines correspond to the cases where the fleet effect does not figure in both the regression and the premium computations (see the numbers in

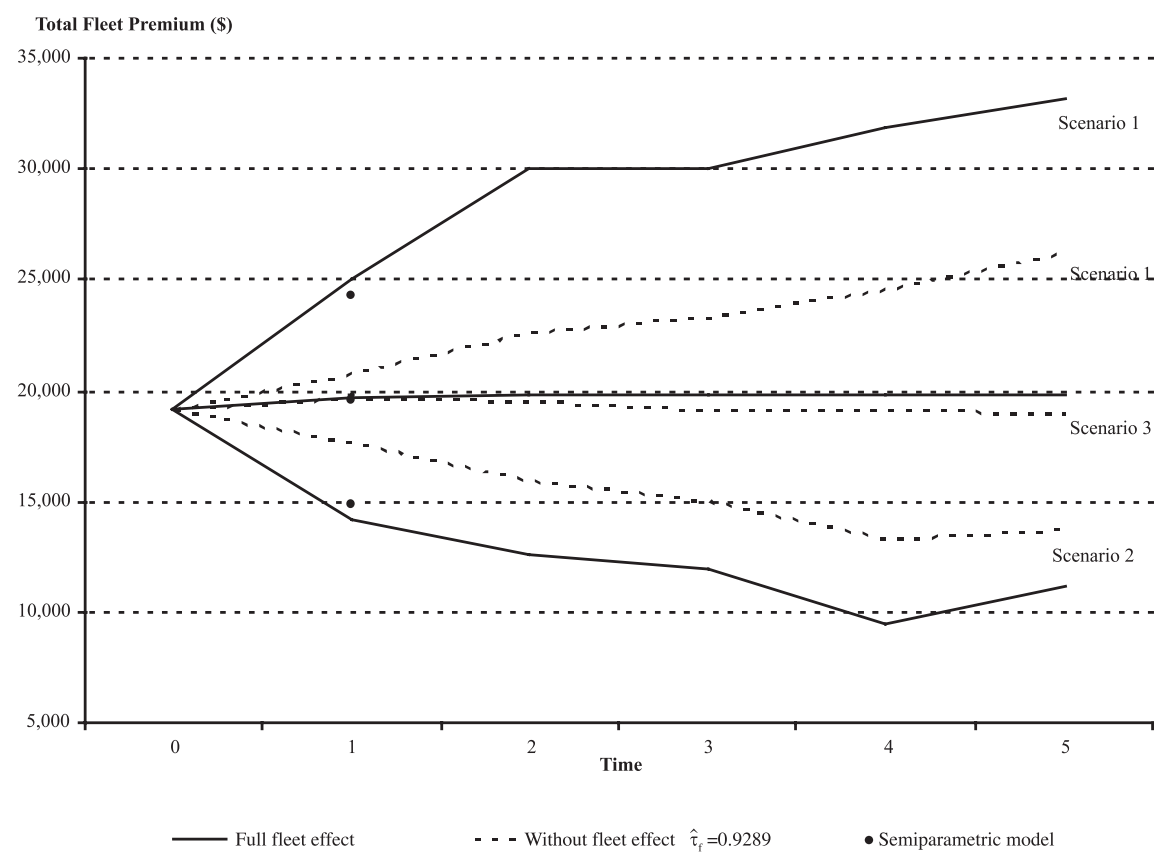

FIGURE 1: Simulation with and without the Fleet Effect. 
TABLE 13

Relative Error for Total Premium Computed BY THE HYPergeOMETRIC MODEL (HH)

\begin{tabular}{l|c|c|c|c}
\hline \hline \multirow{2}{*}{\begin{tabular}{l}
\multirow{2}{*}{$\begin{array}{c}\text { Number } \\
\text { of trucks }\end{array}$} \\
\cline { 3 - 4 }
\end{tabular}} & HMC & HH & \multirow{2}{*}{ Relative error } \\
\hline Simulate fleet & 150 & $\$ 177,712.65$ & $\$ 178,020.34$ & $0.17 \%$ \\
\hline Real fleet & 149 & $\$ 502,498.02$ & $\$ 504,704.59$ & $0.44 \%$ \\
\hline \hline
\end{tabular}

Table 11). The differences are significant. Introducing the fleet effect increases the fluctuations in the premiums and should introduce more incentives for road safety. Finally, we also indicate with solid dots the semiparametric solutions at time 1 . We still observe that the variations in the premiums are lower than in the parametric model developed in this study. The corresponding numbers of the three scenarios are given in Table 12.

Finally, we computed the relative errors in total premium estimation for a large fleet which may be generated when the hypergeometric method is used instead of the Monte Carlo method. We observe from Table 13 that the relative errors are non significant. The notation $\mathrm{HH}$ means that the parameters and the premiums were computed with the hypergeometric method while the notation HMC means that the parameters were estimated with the hypergeometric method, while the premiums were computed with the Monte Carlo method.

\section{CONCLUSION}

In this article, we have developed a parametric model for rating insurance premiums for fleets of vehicles. We have shown how taking into account both fleet and vehicle effects can affect the Bayesian calculation of insurance premiums over time. The model proposed was estimated using data over a single period. An important extension would be to model a panel effect which would take into account the repetitions of information on fleets and vehicles over time (see Abowd et al., 1999, for a first analysis of this type of model, and Angers et al., 2006, for an extension).

We have made comparisons between our hypergeometric model and a Monte Carlo model. For the parameters estimation both models yield the same results but the computing time is much lower with the hypergeometric approximation. For the bonus malus application, both models perform equally but the Monte Carlo is again more time consuming.

We have also compared our hypergeometric model to a semiparametric one. The parametric model permits wider variations in premiums and should introduce more incentive for road safety. Both are equivalent in computing time. 
The rating formula developed presupposes a decentralized management of road safety as regards carriers. In effect, charging different premiums for each of the vehicles in a fleet based on the experience of both the fleet and its trucks will prompt road-safety managers themselves to keep a close eye on road-safety policy and to set up institutional incentives motivating drivers and carriers to adopt prudent behaviors. Indeed, knowing which drivers and carriers are risky, these managers can then assign sliding premiums according to the risk levels of the different drivers and trucks.

\section{ACKNOWLEDGMENT}

A first version of the paper vas presented at the 2004 Risk Theory Seminar in New York City and at the 2004 SCSE meeting in Quebec City. The research was financed by the Programme d'action concertée en sécurité routière FCAR$S A A Q-M T Q$, by the Canada Research Chair in Risk Management, by the Fédération Française des Sociétés d'Assurances, and by the RQCHP. Detailed comments from two referees were very useful in improving both the content and the presentation of the paper. We thank Claire Boisvert very much for her contribution in the preparation of the manuscript.

\section{REFERENCES}

Abowd, J.-M., Kramarz, F., and Margolis, D.N. (1999) High Wage Workers and High Wage Firms. Econometrica 67, 251-333.

Angers, J.F., Desjardins, D., Dionne, G., and Guertin F. (2006) Individual and Firms Random Effects in the Estimation of Event Distributions. Working Paper, Canada Research Chair in Risk Management, HEC Montréal, and CRT, Université de Montréal.

Angers, J.F., Desjardins, D., and Dionne, G. (2004) Modèle bayésien de tarification de l'assurance des flottes de véhicules. L'Actualité économique 80, 253-305.

Dionne, G., Desjardins, D., and Pinquet, J. (1999) L'évaluation du risque d'accident des transporteurs en fonction de leur secteur d'activité, de la taille de leur flotte et de leur dossier d'infractions. Research report 2000-28, Centre de recherche sur les transports, Université de Montréal, $154 \mathrm{p}$.

Dionne, G., Desjardins, D., and Pinquet, J. (2001) Experience Rating Schemes for Fleets of Vehicles. ASTIN Bulletin 31, 85-109.

Dionne, G., Desjardins, D., Ingabire, M.G., and Akdim, R. (2001) La perception du risque d'être arrêté chez les camionneurs et transporteurs routiers. Research report 2001-05, Centre de recherche sur les transports, Université de Montréal, 139 p.

Dionne, G., Laberge-Nadeau, C., Desjardins, D., Messier, S., and Maag, U. (1999) Analysis of the Economic Impact of Medical and Optometric Driving Standards on Costs Incurred by Trucking Firms and on the Social Cost of Traffic Accidents. In Automobile Insurance: Road Safety, New Drivers, Risks, Insurance Fraud and Regulation (ed. G. Dionne and C. LabergeNadeau), pp. 323-351, Kluwer Academic Publishers, Boston.

Dionne, G. and Vanasse, C. (1989) A Generalization of Automobile Insurance Rating Models: The Negative Binomial Distribution with a Regression Component. ASTIN Bulletin 19, 199212.

Dionne, G. and Vanasse, C. (1992) Automobile Insurance Ratemarking in the Presence of Asymmetrical Information. Journal of Applied Econometrics 7, 149-165. 
Frangos, N. and Vrontos, S.D. (2001) Design of Optimal Bonus malus Systems with a Frequency and a Severity Component on an Individual Basis in Automobile Insurance. ASTIN Bulletin 31, 1-22.

FLuet, C. (1999) Commercial Vehicle Insurance: Should Fleet Policies Differ from Single Vehicle Plans? In Automobile Insurance: Road Safety, New Drivers, Risks Insurance Fraud and Regulation (ed. G. Dionne and C. Laberge-Nadeau), 101-117, Kluwer Academic Publishers, Boston.

Gouriéroux, C. (1999) Statistiques de l'assurance. Economica, Paris, 297 p.

Gouriéroux, C., Monfort, A., and Trognon, A. (1984) Pseudo Maximum Likelihood Methods: Application to Poisson Models. Econometrica 52, 701-720.

Gradshteyn, I.S. and Ryzhik, I.M. (1980) Table of Integrals, Series, and Products. Academic Press Inc., New York, 1160 pages.

Hausman, J.A., Hall, B.H., and Griliches, Z. (1984) Econometric Models for Count Data with an Application to the Patents- R\&D Relationship. Econometrica 52, 909-938.

Johnson, N. and Kotz, K. (1969) Discrete Distributions. Houghton Mifflin, Boston, 328 p.

LANGe, K. (1999) Numerical Analysis for Statisticians. Springer: New York, section 21.2, 189-198.

Lemaire, J. (1985) Automobile Insurance: Actuarial Models. Huebner International Series on Risk, Insurance and Economic Security, Kluwer Academic Publishers, Boston, 248 p.

Lemaire, J. (1995) Bonus Malus Systems in Automobile Insurance. Kluwer Academic Publishers, Boston, $283 \mathrm{p}$.

Marie-Jeanne, P. (1994) Problèmes spécifiques des flottes automobiles. Proceedings of the ISUP conference "Cours Avancés sur l'Assurance Automobile."

Moses, L.N. and Savage, I. (1994) The Effect of Firm Characteristics on Truck Accidents. Accident Analysis \& Prevention 26, 173-179.

Moses L.N. and Savage, I. (1996) Identifying Dangerous Trucking Firms. Risk Analysis 16, 359-366.

Pinquet, J. (1997) Allowance for Cost of Claims in Bonus malus Systems. ASTIN Bulletin 27, $23-40$.

Pinquet, J. (1998) Designing Optimal Bonus malus Systems from Different Types of Claims. ASTIN Bulletin 28, 205-220.

Pinquet, J. (2000) Experience Rating through Heterogeneous Models. In Handbook of Insurance, (ed. G. Dionne), Kluwer Academic Publishers, Boston, 459-500.

Purcaru, O. and Denuit, M. (2003) Dependence in Dynamic Claim Frequency Credibility Models. ASTIN Bulletin 33, 23-40.

Teugels, J.L. and Sundt, B. (1991) A Stop-Loss Experience Rating Scheme for Fleets of Cars. Insurance: Mathematics and Economics, North-Holland, 173-179.

Winter, R. (2000) Optimal Insurance under Moral Hazard. In Handbook of Insurance (ed. G. Dionne), Kluwer Academic Publishers, Boston, 155-184.

\section{GEORGES Dionne}

Canada Research Chair in Risk Management,

HEC Montréal, 3000, Chemin de la Côte-Sainte-Catherine,

Montreal (Qc) Canada, H3T 2 A7.

Tel.: (514)340-6596.

Fax: (514)340-5019.

E-mail: georges.dionne@hec.ca.

Jean-François Angers, Denise Desjardins, and François Guertin

Center for Research on Transportation,

Université de Montréal, C.P. 6128, Succ. Centre-Ville,

Montreal, Canada, H3C $3 J 7$. 
The integral to evaluate in equation (8) is given by

$$
\int_{\sum \theta_{f i}=1} \cdots \int \frac{\prod_{i=1}^{I_{f}} \theta_{f i}^{v_{i}+y_{f i}^{j}-1}}{\left(\tau_{f}+\sum_{i=1}^{I_{f}} \theta_{f i} \gamma_{f i}^{j}\right)^{I_{f} \tau_{f}+\sum_{i=1}^{I_{f}} y_{f i}^{j}}} d \theta_{f} .
$$

This integral is almost impossible to do analytically. However, it can be approximated in the following way.

In order to simplify the notation we assumed that $\gamma_{f 1}^{j} \leq \gamma_{f 2}^{j} \leq \ldots, \gamma_{f g}^{j}<\gamma_{f(g+1)}^{j}$ $\leq \ldots \gamma_{f I_{f}}^{j}$. The main idea is to divide $\gamma_{f 1}^{j}, \gamma_{f 2}^{j}, \ldots, \gamma_{f I_{f}}^{j}$ into two subgroups which are as homogenous as possible, that is $\gamma_{f g}^{j}-\gamma_{f 1}^{j}$ and $\gamma_{f I_{f}}^{j}-\gamma_{f(g+1)}^{j}$ are as small as possible. Next, we do the change of variables

$$
\begin{aligned}
& \mu=\sum_{i=1}^{g} \theta_{f i}, \\
& u_{i}=\frac{\theta_{f i}}{\mu} \text { for } i=1,2, \ldots, g-1, \\
& w_{i}=\frac{\theta_{f(i+g)}}{1-\mu} \text { for } i=1, g+2, \ldots, I_{f}-g .
\end{aligned}
$$

The Jacobian of this transformation is given by

$$
|J|=\mu^{g-1}(1-\mu)^{I_{f}-g-1} .
$$

Consequently, the numerator of the integrant of equation (A-1) can be written as

$$
\begin{aligned}
\prod_{i=1}^{I_{f}} \theta_{f i}^{v_{i}+y_{f i}^{j}-1} & =\left[\prod_{i=1}^{g}\left(\mu u_{i}\right)^{v_{i}+y_{f i}^{j}-1}\right]\left[\prod_{i=1}^{I_{f}-g}\left(\{1-\mu\} w_{i}\right)^{v_{i+g}+y_{f(i+g)}^{j}-1}\right] \\
& =\mu^{S_{1}-g}(1-\mu)^{S_{2}-I_{f}+g}\left[\prod_{i=1}^{g} u_{i}^{v_{i}+y_{f i}^{j}-1}\right]\left[\prod_{i=1}^{I_{f}-g}\left(w_{i}\right)^{v_{i+g}+y_{f(i+g)}^{j}-1}\right]
\end{aligned}
$$

where $u_{g}=\theta_{f g} / \mu, S_{1}=\sum_{i=1}^{g}\left(v_{i}+y_{f i}^{j}\right)$ and $S_{2}=\sum_{i=1}^{I_{f}-g}\left(v_{i+g}+y_{f(i+g)}^{j}\right)$. 
Let $\gamma_{(1)}=g^{-1} \sum_{i=1}^{g} \gamma_{f i}^{j}$ and $\gamma_{(2)}=\left(I_{f}-g\right)^{-1} \sum_{i=1}^{I_{f}-g} \gamma_{f(i+g)}^{j}$ and $S_{3}=I_{f} \tilde{t}_{f}+\sum_{i=1}^{I_{f}} y_{f i}^{j}$. Hence, the denominator of equation (A-1) can be written as

$$
\begin{aligned}
& \left(\tau_{f}+\sum_{i=1}^{I_{f}} \theta_{f i} \gamma_{f i}^{j}\right)^{S_{3}} \\
& =\left(\tau_{f}+\sum_{i=1}^{g} \mu u_{i}\left[\gamma_{f i}^{j}-\gamma_{(1)}+\gamma_{(1)}\right]+\sum_{i=1}^{I_{f}-g}(1-\mu) w_{i}\left[\gamma_{f(i+g)}^{j}-\gamma_{(2)}+\gamma_{(2)}\right]\right)^{S_{3}} \\
& =\left(\tau_{f}+\mu \gamma_{(1)} \sum_{i=1}^{g} u_{i}+(1-\mu) \gamma_{(2)} \sum_{i=1}^{I_{f}-g} w_{i}+\mu \sum_{i=1}^{g} u_{i}\left[\gamma_{f i}^{j}-\gamma_{(1)}\right]\right. \\
& \left.\quad+(1-\mu) \sum_{i=1}^{I_{f}-g} w_{i}\left[\gamma_{f(i+g)}^{j}-\gamma_{(2)}\right]\right)^{S_{3}} \\
& =\left(\tau_{f}+\mu \gamma_{(1)}+(1-\mu) \gamma_{(2)}+\mu \sum_{i=1}^{g} u_{i}\left[\gamma_{f i}^{j}-\gamma_{(1)}\right]+(1-\mu) \sum_{i=1}^{I_{f}-g} w_{i}\left[\gamma_{f(i+g)}^{j}-\gamma_{(2)}\right]\right)^{S_{3}} \\
& =\left(\tau_{f}+\mu \gamma_{(1)}+(1-\mu) \gamma_{(2)}\right)^{S_{3}} \\
& \quad \times\left(1+\frac{\mu}{\tau_{f}+\mu \gamma_{(1)}+(1-\mu) \gamma_{(2)}} \sum_{i=1}^{g} u_{i}\left[\gamma_{f i}^{j}-\gamma_{(1)}\right]\right. \\
& \left.\quad+\frac{(1-\mu)}{\tau_{f}+\mu \gamma_{(1)}+(1-\mu) \gamma_{(2)}} \sum_{i=1}^{I_{f}-g} w_{i}\left[\gamma_{f(i+g)}^{j}-\gamma_{(2)}\right]\right)^{S_{3}} .
\end{aligned}
$$

Let $A_{\mu}=\mu /\left(\tau_{f}+\mu \gamma_{(1)}+(1-\mu) \gamma_{(2)}\right), B_{\mu}=(1-\mu) /\left(\tau_{f}+\mu \gamma_{(1)}+(1-\mu) \gamma_{(2)}\right)$ and

$$
g(u, w)=\left(1+A_{v} \sum_{i=1}^{g} u_{i}\left[\gamma_{f i}^{j}-\gamma_{(1)}\right]+B_{v} \sum_{i=1}^{I_{f}-g} w_{i}\left[\gamma_{f i}^{j}-\gamma_{(2)}\right]\right)^{-S_{3}} .
$$

Expanding $g(\underset{\sim}{u}, w)$ in a Taylor series of order 1 around $u_{0}=(1 / g, 1 / g, \ldots, 1 / g)^{t}$ and $w_{0}=\left(1 /\left(I_{f}-g\right), 1 /\left(I_{f}-g\right), \ldots, 1 /\left(I_{f}-g\right)\right)^{t}, g(u, w)$ can be written as

$$
\begin{aligned}
g(\underset{\sim}{u}, \mathcal{W})=1-S_{3} A_{\mu} \sum_{i=1}^{g}\left(u_{i}^{*}-1 / g\right)\left(\gamma_{f i}^{j}-\gamma_{f g}^{j}\right) & \\
& -S_{3} B_{\mu} \sum_{i=1}^{I_{f}-g}\left(w_{i}^{*}-1 /\left[I_{f}-g\right]\right)\left(\gamma_{f(i+g)}^{j}-\gamma_{f I_{f}}^{j}\right) .
\end{aligned}
$$

where $u^{*}$ and $w^{*}$ are points between the boundary of the simplex and $u_{0}$ and $w_{0}$. Using equations (A-2), (A-3), (A-4) and (A-5), equation (A-1) can be written as 


$$
\begin{aligned}
& \int_{\Sigma \theta_{f i}=1} \cdots \int \frac{\prod_{i=1}^{I_{f}} \theta_{f i}^{v_{i}+y_{f i}^{\prime}-1}}{\left(\tau_{f}+\sum_{i=1}^{I_{f}} \theta_{f i} \gamma_{f i}^{j}\right)^{I_{f} \kappa_{f}^{-1}+\sum_{i=1}^{I_{f}} y_{f i}^{j}} d \theta_{f}} \\
& =\int_{\Sigma \theta_{f i}=1} \cdots \int \frac{\mu^{S_{1}-g}(1-\mu)^{S_{2}-I_{f}+g}\left[\prod_{i=1}^{g} u_{i}^{v_{i}+y_{f i}^{j}-1}\right]\left[\prod_{i=1}^{I_{f}-g}\left(w_{i}\right)^{v_{i+g}+y_{f(i+g)}^{j}-1}\right]}{\left(\tau_{f}+\mu \gamma_{(1)}+(1-\mu) \gamma_{(2)}\right)^{S_{3}}} \\
& \left.-S_{3} B_{\mu} \sum_{i=1}^{I_{f}-g}\left(w_{i}^{*}-1 /\left[I_{f}-g\right]\right)\left(\gamma_{f(i+g)}^{j}-\gamma_{f I_{f}}^{j}\right)\right] \mu^{g-1}(1-\mu)^{I_{f}-g-1} d \underset{\sim}{g}\left(u_{i}^{*}-1 / g\right)\left(\gamma_{f i}^{j}-\gamma_{f g}^{j}\right)
\end{aligned}
$$$$
=\int_{\sum \theta_{f i}=1} \cdots \int \frac{\mu^{S_{1}-1}(1-\mu)^{S_{2}-1}\left[\prod_{i=1}^{g} u_{i}^{v_{i}+y_{f i}^{j}-1}\right]\left[\prod_{i=1}^{I_{f}-g}\left(w_{i}\right)^{v_{i+g}+y_{f(i+g)}^{j}-1}\right]}{\left(\tau_{f}+\mu \gamma_{(1)}+(1-\mu) \gamma_{(2)}\right)^{S_{3}}}
$$$$
\left[1-S_{3} \frac{\mu}{\left(\tau_{f}+\mu \gamma_{(1)}+(1-\mu) \gamma_{(2)}\right)} \sum_{i=1}^{g}\left(u_{i}^{*}-1 / g\right)\left(\gamma_{f i}^{j}-\gamma_{f g}^{j}\right)\right.
$$$$
\left.-S_{3} \frac{(1-\mu)}{\left(\tau_{f}+\mu \gamma_{(1)}+(1-\mu) \gamma_{(2)}\right)} \sum_{i=1}^{I_{f}-g}\left(w_{i}^{*}-1 /\left[I_{f}-g\right]\right)\left(\gamma_{f(i+g)}^{j}-\gamma_{f I_{f}}^{j}\right)\right] d \underset{\sim}{u} d \underset{\sim}{w} d \mu
$$$$
=\frac{\prod_{i=1}^{I_{f}} \Gamma\left(y_{f i}^{j}+v_{i}\right)}{\Gamma\left(S_{1}\right) \Gamma\left(S_{2}\right)}\left[\int_{\Sigma \theta_{f i}=1} \cdots \int \frac{\mu^{S_{1}-1}(1-\mu)^{S_{2}-1}}{\left(\tau_{f}+\mu \gamma_{(1)}+(1-\mu) \gamma_{(2)}\right)^{S_{3}}} d \mu\right.
$$$$
-S_{3} \sum_{i=1}^{g}\left(u_{i}^{*}-1 / g\right)\left(\gamma_{f i}^{j}-\gamma_{f g}^{j}\right) \int_{\Sigma \theta_{f i}=1} \cdots \int \frac{\mu^{S_{1}}(1-\mu)^{S_{2}-1}}{\left(\tau_{f}+\mu \gamma_{(1)}+(1-\mu) \gamma_{(2)}\right)^{S_{3}+1}} d \mu
$$$$
\left.-S_{3} \sum_{i=1}^{I_{f}-g}\left(w_{i}^{*}-1 /\left[I_{f}-g\right]\right)\left(\gamma_{f(i+g)}^{j}-\gamma_{f g}^{j}\right) \int_{\Sigma \theta_{f i}=1} \cdots \int \frac{\mu^{S_{1}-1}(1-\mu)^{S_{2}}}{\left(\tau_{f}+\mu \gamma_{(1)}+(1-\mu) \gamma_{(2)}\right)^{S_{3}+1}} d \mu\right] \text {. }
$$

Using an increasing factorial function (Gradshteyn and Ryzhik, 1980, Section 9.1), we then have

$$
\begin{aligned}
& \int_{\Sigma \theta_{f i}=1} \cdots \int \frac{\prod_{i=1}^{I_{f}} \theta_{f i}^{v_{i}+y_{f i}^{j}-1}}{\left(\tau_{f}+\sum_{i=1}^{I_{f}} \theta_{f i} \gamma_{f i}^{j}\right)^{I_{f} \kappa_{f}^{-1}+\sum_{i=1}^{I_{f}} y_{f i}^{j}} d \theta_{f}} \\
& =\frac{\prod_{i=1}^{I_{f}} \Gamma\left(y_{f i}^{j}+v_{i}\right)}{\Gamma\left(S_{1}\right) \Gamma\left(S_{2}\right)}\left[\frac{\Gamma\left(S_{1}\right) \Gamma\left(S_{2}\right)}{\left(\tau_{f}+\gamma_{(2)}\right)^{S_{3}} \Gamma\left(S_{1}+S_{2}\right)}{ }_{2} F_{1}\left(S_{1} ; S_{3} ; S_{1}+S_{2} ; \frac{\gamma_{(2)}-\gamma_{(1)}}{\kappa^{-1}+\gamma_{(2)}}\right)\right.
\end{aligned}
$$




$$
\begin{aligned}
& -S_{3} \sum_{i=1}^{g}\left(u_{i}^{*}-1 / g\right)\left(\gamma_{f i}^{j}-\gamma_{f g}^{j}\right) \frac{\Gamma\left(S_{1}+1\right) \Gamma\left(S_{2}\right)}{\left(\tau_{f}+\gamma_{(2)}\right)^{S_{3}+1} \Gamma\left(S_{1}+S_{2}+1\right)} \\
& \times{ }_{2} F_{1}\left(S_{1}+1 ; S_{3}+1 ; S_{1}+S_{2}+1 ; \frac{\gamma_{(2)}-\gamma_{(1)}}{\tau_{f}+\gamma_{(2)}}\right) \\
& -S_{3} \sum_{i=1}^{I_{f}-g}\left(w_{i}^{*}-1 /\left[I_{f}-g\right]\right)\left(\gamma_{f(i+g)}^{j}-\gamma_{f g}^{j}\right) \frac{\Gamma\left(S_{1}\right) \Gamma\left(S_{2}+1\right)}{\left(\tau_{f}+\gamma_{(2)}\right)^{S_{3}+1} \Gamma\left(S_{1}+S_{2}+1\right)} \\
& \left.\times{ }_{2} F_{1}\left(S_{1} ; S_{3}+1 ; S_{1}+S_{2}+1 ; \frac{\gamma_{(2)}-\gamma_{(1)}}{\tau_{f}+\gamma_{(2)}}\right)\right] \\
& =\frac{\prod_{i=1}^{I_{f}} \Gamma\left(y_{f i}^{j}+v_{i}\right)}{\left(\tau_{f}+\gamma_{(2)}\right)^{S_{3}} \Gamma\left(S_{1}+S_{2}\right)}{ }_{2} F_{1}\left(S_{1} ; S_{3} ; S_{1}+S_{2} ; \frac{\gamma_{(2)}-\gamma_{(1)}}{\tau_{f}+\gamma_{(2)}}\right) \\
& {\left[1-\left\{\sum_{i=1}^{g}\left(u_{i}^{*}-1 / g\right)\left(\gamma_{f i}^{j}-\gamma_{f g}^{j}\right)\right\} \frac{S_{1} S_{3}}{\left(\tau_{f}+\gamma_{(2)}\right)\left(S_{1}+S_{2}\right)}\right.} \\
& \times \frac{{ }_{2} F_{1}\left(S_{1}+1 ; S_{3}+1 ; S_{1}+S_{2}+1 ; \frac{\gamma_{(2)}-\gamma_{(1)}}{\tau_{f}+\gamma_{(2)}}\right)}{{ }_{2} F_{1}\left(S_{1} ; S_{3} ; S_{1}+S_{2} ; \frac{\gamma_{(2)}-\gamma_{(1)}}{\tau_{f}+\gamma_{(2)}}\right)} \\
& -\left\{\sum_{i=1}^{I_{f}-g}\left(w_{i}^{*}-1 /\left[I_{f}-g\right]\right)\left(\gamma_{f i}^{j}-\gamma_{f I_{f}}^{j}\right)\right\} \frac{S_{2} S_{3}}{\left(\tau_{f}+\gamma_{(2)}\right)\left(S_{1}+S_{2}\right)} \\
& \left.\times \frac{{ }_{2} F_{1}\left(S_{1} ; S_{3}+1 ; S_{1}+S_{2}+1 ; \frac{\gamma_{(2)}-\gamma_{(1)}}{\tau_{f}+\gamma_{(2)}}\right)}{{ }_{2} F_{1}\left(S_{1} ; S_{3} ; S_{1}+S_{2} ; \frac{\gamma_{(2)}-\gamma_{(1)}}{\tau_{f}+\gamma_{(2)}}\right)}\right] .
\end{aligned}
$$

To illustrate the relative precision of this approximation, we computed the average of the expression in squared bracket of the last equation for several values of $I_{f}$ and $g$. To do so, we selected $I_{f}$ values at random among the following values of $\gamma_{f i}^{j}$ :

$\{0.00929,0.01316,0.01734,0.01862,0.01952,0.02065,0.02223,0.03725,0.04527$, $0.04998,0.05643,0.05785,0.06076,0.09432,0.10037,0.10771,0.10800,0.10893$, $0.11646,0.11935,0.12243,0.12864,0.13466,0.13469,0.14709,0.14724,0.14781$, $0.14998,0.15502,0.15788,0.16524,0.17454,0.18125,0.18470,0.20312,0.20995$, $0.21316,0.21316,0.21626,0.21760,0.22727,0.24248,0.24624,0.25641,0.27592$, $0.29423,0.30632,0.32179,0.32457,0.44040\}$,

along with $\tau_{f}=0.1542$ and $v=2.2056$. We also generate $\max \left\{1,0.1 \times I_{f}\right\}$ accidents at random with probability proportional to the $\gamma_{f i}^{j}$. The value of $g$ was 
chosen in order to minimize $\left(V_{1}+V_{2}\right) / V$ where $V_{i}$ is the standard deviation of the $\gamma_{f i}^{j}$ for the elements of the $i^{\text {th }}$ group and $V$, the standard deviation of the $\gamma_{f i}^{j}$ for the whole fleet.

In the following figure, we plotted the relative error as a function of $I_{f}$.

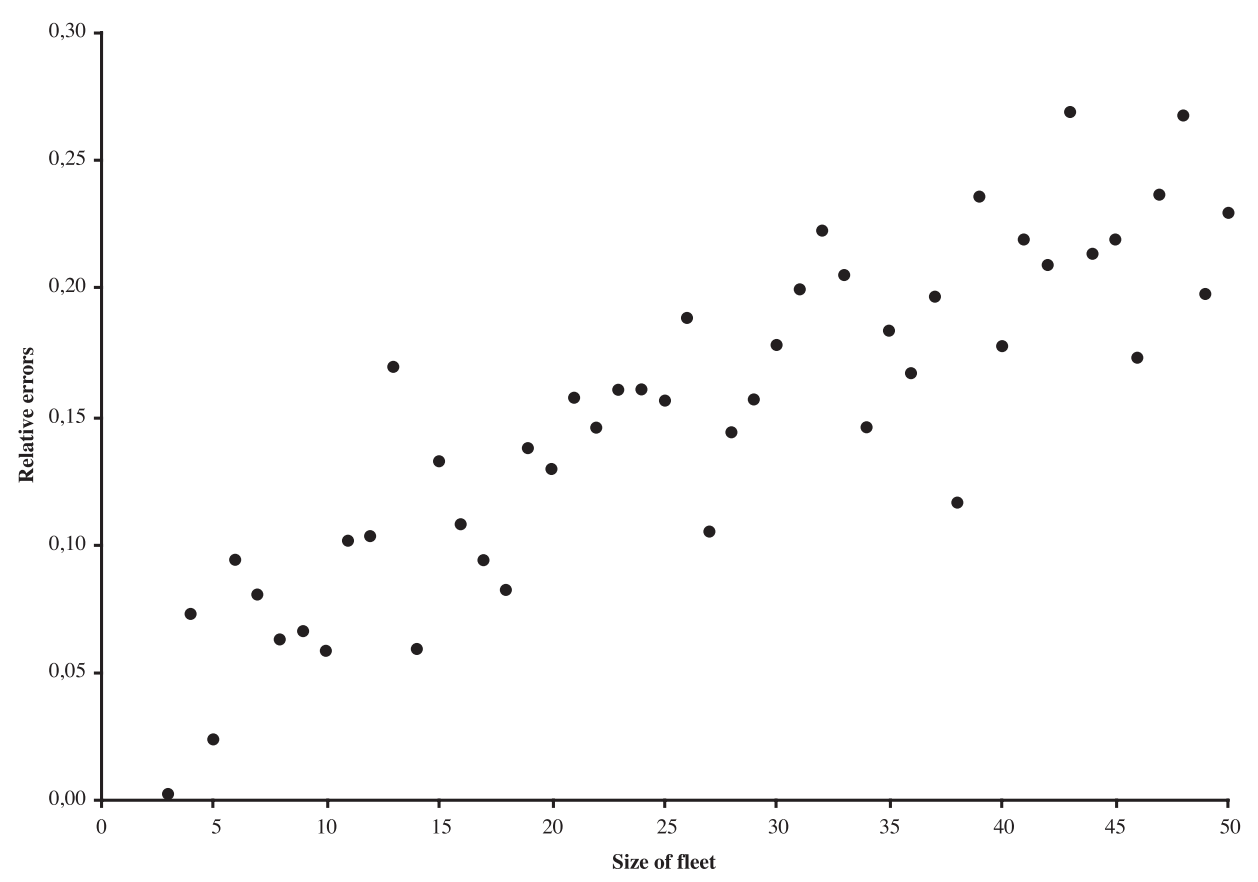

FIGURE 2: Relative Errors as a Function of Size of Fleet.

As expected, the relative error increases as $I_{f}$ increases. This can be explained by the fact that the more trucks there are in a fleet, the more likely it is to see some variation between the $\gamma_{f i}^{j}$. The correlation between $I_{f}$ and the relative error is around 0.88 . 
- APPENDIX B -

GRADIENT AND HESSIAN COMPUTATION

For matters of faster convergence, we made the following variable change: $\hat{\tau}_{f}=$ $\kappa_{f}^{-1}$. The Log-likelihood function of equation (11) for $F$ firms is equal to:

$$
\begin{aligned}
L=\sum_{f=1}^{F} & {\left[\log \Gamma\left(I_{f} \kappa_{f}^{-1}+\sum_{i=1}^{I_{f}} y_{f i}^{j}\right)-\log \Gamma\left(I_{f} \kappa_{f}^{-1}\right)+\left(\sum_{i=1}^{I_{f}} y_{f i}^{j}\right) \times \log \left(\kappa_{f}\right)-\right.} \\
& \left(I_{f} \kappa_{f}^{-1}+\sum_{i=1}^{I_{f}} y_{f i}^{j}\right) \log \left(1+\kappa_{f} \gamma_{f g 2}\right)+ \\
& \log \Gamma\left(\sum_{i=1}^{I_{f}} v_{i}\right)-\log \Gamma\left(\sum_{i=1}^{I_{f}}\left(v_{i}+y_{f i}^{j}\right)\right)+\sum_{i=1}^{I_{f}} \log \Gamma\left(v_{i}+y_{f i}^{j}\right)+ \\
& \sum_{i=1}^{I_{f}} y_{f i}^{j} \log \left(\gamma_{f i}^{j}\right)-\sum_{i=1}^{I_{f}} \log \Gamma\left(v_{i}\right)-\sum_{i=1}^{I_{f}} \log \Gamma\left(y_{f i}^{j}+1\right)+ \\
& \left.\log \left({ }_{2} F_{1}\left(\sum_{i=1}^{g}\left(v_{i}+y_{f i}^{j}\right), I_{f} \kappa_{f}^{-1}+\prod_{i=1}^{I_{f}} y_{f i}^{j}, \sum_{i=1}^{I_{f}}\left(v_{i}+y_{f i}^{j}\right),\left(\frac{\gamma_{f g 2}-\gamma_{f g 1}}{\kappa_{f}^{-1}+\gamma_{f g 2}}\right)\right)\right)\right] .
\end{aligned}
$$

We used the maximum likelihood method to estimate the unknown parameters, $v, \kappa_{f}, \beta=\beta_{1}, \ldots, \beta_{m}$. The gradient and Hessian take the following forms:

\section{Gradient}

$$
\begin{aligned}
\frac{\partial L}{\partial v_{i}}= & \sum_{f=1}^{F} \frac{\partial L_{f}}{\partial v_{i}} \\
\frac{\partial L_{f}}{\partial v_{i}}= & \Psi\left(\sum_{i=1}^{I_{f}} v_{i}\right)-\Psi\left(\sum_{i=1}^{I_{f}}\left(v_{i}+y_{f i}^{j}\right)\right)+\sum_{i=1}^{I_{f}} \Psi\left(v_{i}+y_{f i}^{j}\right)-\sum_{i=1}^{I_{f}} \Psi\left(v_{i}\right) \\
& +\frac{\partial}{\partial v_{i}} \log \left({ }_{2} F_{1}(a, b, c, d)\right)
\end{aligned}
$$

with $\Psi()=.\frac{\Gamma^{\prime}(.)}{\Gamma(.)}$ denotes the first derivative of the natural logarithm function of gamma function $\Gamma\left(\right.$.) and its derivative $\Gamma^{\prime}($.) and where:

$$
\begin{array}{ll}
a=\sum_{i=1}^{I_{f}} \chi_{1 i}\left(v_{i}+y_{f i}^{j}\right) & b=\left(I_{f} \kappa_{f}^{-1}+\sum_{i=1}^{I_{f}} y_{f i}^{j}\right) \\
c=\sum_{i=1}^{I_{f}}\left(v_{j}+y_{f i}^{j}\right) & d=\left(\frac{\gamma_{f g 2}-\gamma_{f g 1}}{\kappa_{f}^{-1}+\gamma_{f g 2}}\right)
\end{array}
$$


and

$\frac{\partial}{\partial v_{i}} \log \left({ }_{2} F_{1}(a, b, c, d)\right) \approx$
$\frac{\log \left({ }_{2} F_{1}\left(\left(v_{i}+y_{f i}^{j}+h_{i}\right)+\sum_{k \neq i}^{I_{f}} \chi_{1 k}\left(y_{f k}^{j}+v_{k}\right), b,\left(v_{i}+y_{f i}^{j}+h_{i}\right)+\sum_{k \neq i}^{I_{f}}\left(v_{k}+y_{f k}^{j}\right), d\right)\right)-\log \left({ }_{2} F_{1}(a, b, c, d)\right)}{h_{i}}$

$\frac{\partial L}{\partial \kappa_{f}}=\sum_{f=1}^{F} \frac{\partial L_{f}}{\partial \kappa_{f}}$

$\frac{\partial L_{f}}{\partial \kappa_{f}}=\kappa_{f}^{-1} \sum_{i=1}^{I_{f}} y_{f i}^{j}+I_{f} \kappa_{f}^{-2} \log \left(1+\kappa_{f} \gamma_{f g 2}\right)-\frac{\left(I_{f} \kappa_{f}^{-1}+\sum_{i=1}^{I_{f}} y_{f i}^{j}\right) \gamma_{f g 2}}{1+\kappa_{f} \gamma_{f g 2}}-$

$$
I_{f} \kappa_{f}^{-2} \Psi\left(I_{f} \kappa_{f}^{-1}+\sum_{i=1}^{I_{f}} y_{f i}^{j}\right)+I_{f} \kappa_{f}^{-2} \Psi\left(I_{f} \kappa_{f}^{-1}\right)+\frac{\partial}{\partial \kappa_{f}} \log \left({ }_{2} F_{1}(a, b, c, d)\right)
$$

$\frac{\partial}{\partial \kappa_{f}} \log \left({ }_{2} F_{1}(a, b, c, d)\right) \approx$

$\frac{\log \left({ }_{2} F_{1}\left(a,\left(I_{f}\left(\kappa_{f}+h_{f}\right)^{-1}+\sum_{i=1}^{I_{f}} y_{f i}^{j}\right), c,\left(\frac{\gamma_{f g 2}-\gamma_{f g 1}}{\left(\kappa_{f}+h_{f}\right)^{-1}+\gamma_{f g 2}}\right)\right)\right)-\log \left({ }_{2} F_{1}(a, b, c, d)\right)}{h_{f}}$

$\frac{\partial L}{\partial \beta}=\sum_{f=1}^{F} \frac{\partial L_{f}}{\partial \beta}$

$\frac{\partial L_{f}}{\partial \beta}=X^{\prime} * Y-\left(I_{f} \kappa_{f}^{-1}+\sum_{i=1}^{I_{f}} y_{f i}^{j}\right)\left(\frac{\kappa_{f}}{1+\kappa_{f} \gamma_{f g 2}}\right)\left(\frac{X^{\prime}}{I_{f}-g} *(\gamma \#\right.$ group $\left.)\right)+\frac{\partial}{\partial \beta} \log \left({ }_{2} F_{1}(a, b, c, d)\right)$

where

$\frac{\partial}{\partial \beta} \log \left({ }_{2} F_{1}(a, b, c, d)\right)=\frac{1}{{ }_{2} F_{1}(a, b, c, d)} \times \frac{\partial}{\partial \beta}{ }_{2} F_{1}(a, b, c, d)$

with

$\frac{\partial}{\partial \beta}{ }_{2} F_{1}(a, b, c, d)={ }_{2} F_{1}(1+a, 1+b, 1+c, d) \times$

$\frac{\sum_{i=1}^{I_{f}} \chi_{1 i}\left(v_{i}+y_{f i}^{j}\right) \times\left(I_{f} \kappa_{f}^{-1}+\sum_{i=1}^{I_{f}} y_{f i}^{j}\right)}{\sum_{i=1}^{I_{f}}\left(v_{i}+y_{f i}^{j}\right)} \times$

$\left[\left(\frac{\frac{X^{\prime}}{I_{f}-g} *(\gamma \# \text { group })-\frac{X^{\prime}}{g} *(\gamma \#(1-\text { group }))}{\kappa_{f}^{-1}+\gamma_{f g 2}}\right)-\frac{\left(\gamma_{f g 2}-\gamma_{f g 1}\right)}{\left(\kappa_{f}^{-1}+\gamma_{f g 2}\right)^{2}} *\left(\frac{X^{\prime}}{I_{f}-g} *(\gamma \#\right.\right.$ group $\left.\left.)\right)\right]$ 
where $X=\left(\begin{array}{c}X_{f 1} \\ \ldots \\ X_{f i} \\ \ldots \\ X_{f I_{f}}\end{array}\right)$ with $X_{f i}=\left(x_{f i 1}, \ldots, x_{f i p}\right) \quad Y=\left(\begin{array}{c}Y_{f 1} \\ \ldots \\ Y_{f i} \\ \ldots \\ Y_{f I_{f}}\end{array}\right) \quad \gamma=\left(\begin{array}{c}\gamma_{f 1} \\ \ldots \\ \gamma_{f i} \\ \ldots \\ \gamma_{f I_{f}}\end{array}\right)$

with $\gamma_{f i}=d_{f i} e^{X_{f i} \beta}$. The group vector of dimension $I_{f}$ corresponds to 0 if truck $i$ is in the first group and to 1 if truck $i$ is in the second group (higher risks).

In matricial notation,

- * corresponds to the matrices multiplication;

- \# corresponds to the elementwise multiplication;

- group $=1$ when the truck is a high risk and 0 otherwise.

\section{Hessian}

$$
\begin{aligned}
& \frac{\partial^{2} L}{\partial v_{q} \partial v_{k}}=\sum_{f=1}^{F} \frac{\partial^{2} L_{f}}{\partial v_{q} \partial v_{k}} \\
& \frac{\partial^{2} L_{f}}{\partial v_{q} \partial v_{q}}=\Psi^{\prime}\left(\sum_{i=1}^{I_{f}} v_{i}\right)-\Psi^{\prime}\left(\sum_{i=1}^{I_{f}}\left(v_{i}+y_{f i}^{j}\right)\right)+\sum_{i=1}^{I_{f}} \Psi^{\prime}\left(v_{i}+y_{f i}^{j}\right)-\sum_{i=1}^{I_{f}} \Psi^{\prime}\left(v_{i}\right)+ \\
& \frac{\partial^{2}}{\partial v_{q} \partial v_{q}} \log \left({ }_{2} F_{1}(a, b, c, d)\right) \\
& \text { with } \frac{\partial^{2}}{\partial v_{q} \partial v_{q}} \log \left({ }_{2} F_{1}(a, b, c, d)\right)= \\
& \frac{\frac{\partial}{\partial v_{q}} \log \left({ }_{2} F_{1}\left(a+h_{q}, b, c+h_{q}, d\right)\right)-\frac{\partial}{\partial v_{q}} \log \left({ }_{2} F_{1}(a, b, c, d)\right)}{h_{q}} \\
& \text { and } \frac{\partial^{2} L}{\partial v_{q} \partial v_{k}}=\Psi^{\prime}\left(\sum_{i=1}^{I_{f}} v_{i}\right)-\Psi^{\prime}\left(\sum_{i=1}^{I_{f}}\left(v_{i}+y_{f i}^{j}\right)\right)+\sum_{i=1}^{I_{f}} \Psi^{\prime}\left(v_{i}+y_{f i}^{j}\right)-\sum_{i=1}^{I_{f}} \Psi^{\prime}\left(v_{i}\right)+ \\
& \frac{\partial^{2}}{\partial v_{q} \partial v_{k}} \log \left({ }_{2} F_{1}(a, b, c, d)\right) \\
& \text { with } \frac{\partial^{2}}{\partial v_{q} \partial v_{k}} \log \left({ }_{2} F_{1}(a, b, c, d)\right)= \\
& \frac{\frac{\partial}{\partial v_{q}} \log \left({ }_{2} F_{1}\left(a+h_{k}, b, c+h_{k}, d\right)\right)-\frac{\partial}{\partial v_{q}} \log \left({ }_{2} F_{1}(a, b, c, d)\right)}{h_{k}}
\end{aligned}
$$

with $\Psi^{\prime}$ denotes the second derivative of the natural logarithm function of gamma function 


$$
\begin{aligned}
\frac{\partial^{2} L}{\partial \kappa_{f} \partial \kappa_{f}} & =\sum_{f=1}^{F} \frac{\partial^{2} L_{f}}{\partial \kappa_{f} \partial \kappa_{f}} \\
\frac{\partial^{2} L_{f}}{\partial \kappa_{f} \partial \kappa_{f}} & =-\kappa_{f}^{-2} \sum_{i=1}^{I_{f}} y_{f i}^{j}-2 I_{f} \kappa_{f}^{-3} \times \log \left(1+\kappa_{f} \gamma_{f g 2}\right)+\frac{\left(I_{f} \kappa_{f}^{-1}+\sum_{i=1}^{I_{f}} y_{f i}^{j}\right)\left(\gamma_{f g 2}\right)^{2}}{\left(1+\kappa_{f} \gamma_{f g 2}\right)^{2}}+ \\
& \frac{2 I_{f} \kappa_{f}^{-2} \gamma_{f g 2}}{1+\kappa_{f} \gamma_{f g 2}}+\left(I_{f} \kappa_{f}^{-2}\right)^{2} \Psi^{\prime}\left(I_{f} \kappa_{f}^{-1}+\sum_{i=1}^{I_{f}} y_{f i}^{j}\right)+2 I_{f} \kappa_{f}^{-3} \Psi\left(I_{f} \kappa_{f}^{-1}+\sum_{i=1}^{I_{f}} y_{f i}^{j}\right)- \\
& \left(I_{f} \kappa_{f}^{-2}\right)^{2} \Psi^{\prime}\left(I_{f} \kappa_{f}^{-1}\right)-2 I_{f} \kappa_{f}^{-3} \Psi\left(I_{f} \kappa_{f}^{-1}\right)+\frac{\partial^{2}}{\partial \kappa_{f} \partial \kappa_{f}} \log \left({ }_{2} F_{1}(a, b, c, d)\right)
\end{aligned}
$$

with

$$
\begin{aligned}
& \frac{\partial^{2}}{\partial \kappa_{f} \partial \kappa_{f}} \log \left({ }_{2} F_{1}(a, b, c, d)\right) \approx \\
& \frac{\frac{\partial}{\partial \kappa_{f}} \log \left({ }_{2} F_{1}\left(a,\left(I_{f}\left(\kappa_{f}+h_{f}\right)^{-1}+\sum_{i=1}^{I_{f}} y_{f i}^{j}\right), c,\left(\frac{\gamma_{f g 2}-\gamma_{f g 1}}{\left(\kappa_{f}+h_{f}\right)^{-1}+\gamma_{f g 2}}\right)\right)\right)-\frac{\partial}{\partial \kappa_{f}} \log \left({ }_{2} F_{1}(a, b, c, d)\right)}{h_{f}}
\end{aligned}
$$

and $\frac{\partial^{2} L_{f}}{\partial \kappa_{f} \partial \kappa_{s}}=0$

$\frac{\partial^{2} L}{\partial v_{j} \partial \kappa_{f}}=\sum_{f=1}^{F} \frac{\partial^{2} L_{f}}{\partial v_{j} \partial \kappa_{f}}$

$\frac{\partial^{2} L_{f}}{\partial v_{i} \partial \kappa_{f}}=$

$\frac{\frac{\partial}{\partial v_{i}} \log \left({ }_{2} F_{1}\left(a,\left(\bar{I}_{f}\left(\kappa_{f}+h_{f}\right)^{-1}+\sum_{i=1}^{I_{f}} y_{f i}^{j}\right), c,\left(\frac{\gamma_{f g 2}-\gamma_{f g 1}}{\left(\kappa_{f}+h_{f}\right)^{-1}+\gamma_{f g 2}}\right)\right)\right)-\frac{\partial}{\partial v_{i}} \log \left({ }_{2} F_{1}(a, b, c, d)\right)}{h_{f}}$

$$
\begin{aligned}
\frac{\partial^{2} L}{\partial v_{i} \partial \beta} & =\sum_{f=1}^{F} \frac{\partial^{2} L_{f}}{\partial v_{i} \partial \beta} \\
\frac{\partial^{2} L_{f}}{\partial v_{i} \partial \beta} & =\frac{\partial^{2}}{\partial v_{i} \partial \beta} \log \left({ }_{2} F_{1}(a, b, c, d)\right) \\
\frac{\partial^{2} L}{\partial \kappa_{f} \partial \beta} & =\sum_{f=1}^{F} \frac{\partial^{2} L_{f}}{\partial \kappa_{f} \partial \beta} \\
\frac{\partial^{2} L_{f}}{\partial \kappa_{f} \partial \beta} & =\left(\frac{I_{f} \kappa_{f}^{-1}}{1+\kappa_{f} \gamma_{f g 2}}\right) *\left(\frac{X^{\prime} *(\gamma \# \text { group })}{I_{f}-g}\right)-\left(\frac{I_{f} \kappa_{f}^{-1}+\sum_{i=1}^{I_{f}} y_{f i}^{j}}{1+\kappa_{f} \gamma_{f g 2}}\right) *\left(\frac{X^{\prime} *(\gamma \# \text { group })}{I_{f}-g}\right)+
\end{aligned}
$$




$$
\left(\frac{I_{f} \kappa_{f}^{-1}+\sum_{i=1}^{I_{f}} y_{f i}^{j}}{\left(1+\kappa_{f} \gamma_{f g 2}\right)^{2}}\right) \frac{\kappa_{f} \gamma_{f g 2}}{I_{f}-g}\left(X^{\prime} *(\gamma \# \text { group })\right)+\frac{\partial^{2} \log \left({ }_{2} F_{1}(a, b, c, d)\right)}{\partial \kappa_{f} \partial \beta}
$$

$\frac{\partial^{2} L}{\partial \beta \partial \beta}=\sum_{f=1}^{F} \frac{\partial L_{f}}{\partial \beta \partial \beta}$

$$
\begin{aligned}
\frac{\partial^{2} L_{f}}{\partial \beta \partial \beta}=- & \left(I_{f} \kappa_{f}^{-1}+\sum_{i=1}^{I_{f}} y_{f i}^{j}\right)\left(\frac{\kappa_{f}}{1+\kappa_{f} \gamma_{f g 2}}\right)\left(\frac{X^{\prime}}{I_{f}-g} *(X \#(\gamma \# \text { group }))\right)+ \\
& \left(I_{f} \kappa_{f}^{-1}+\sum_{i=1}^{I_{f}} y_{f i}^{j}\right)\left(\frac{\kappa_{f}}{1+\kappa_{f} \gamma_{f g 2}}\right)^{2}\left(\frac{X^{\prime}}{I_{f}-g} *((\gamma \# \text { group }))\right) * \\
& \left(\frac{X^{\prime}}{I_{f}-g} *((\gamma \# \text { group }))\right)^{\prime}+\frac{\partial^{2} \log \left({ }_{2} F_{1}(a, b, c, d)\right)}{\partial \beta \partial \beta}
\end{aligned}
$$

with

$$
\begin{aligned}
& \frac{\partial^{2} \log \left({ }_{2} F_{1}(a, b, c, d)\right)}{\partial \beta \partial \beta}= \\
& \quad\left[c c \times c c 1 \times \frac{{ }_{2} F_{1}(2+a, 2+b, 2+c, d)}{{ }_{2} F_{1}(a, b, c, d)}-c c^{2}\left(\frac{{ }_{2} F_{1}(1+a, 1+b, 1+c, d)}{{ }_{2} F_{1}(a, b, c, d)}\right)^{2}\right] \times W^{2}+ \\
& \quad\left[c c \times \frac{{ }_{2} F_{1}(1+a, 1+b, 1+c, d)}{{ }_{2} F_{1}(a, b, c, d)}\right] \frac{\partial W}{\partial \beta}
\end{aligned}
$$

with

$$
\begin{aligned}
& c c=\frac{\sum_{i=1}^{I_{f}} \chi_{1 i}\left(v_{i}+y_{f i}^{j}+\right) \times\left(I_{f} \kappa_{f}^{-1}+\sum_{i=1}^{I_{f}} y_{f i}^{j}\right)}{\sum_{i=1}^{I_{f}}\left(v_{i j}+y_{f i}^{j}\right)} \\
& c c 1=\frac{\left(1+\sum_{i=1}^{I_{f}}\left(v_{i}+y_{f i}^{j}\right)\right) \times\left(1+I_{f} \kappa_{f}^{-1}+\sum_{i=1}^{I_{f}} y_{f i}^{j}\right)}{1+\sum_{i=1}^{I_{f}}\left(v_{i}+y_{f i}^{j}\right)} \\
& W=\left(\frac{\frac{X^{\prime}}{I_{f}-g} *(\gamma \# \text { group })-\frac{X^{\prime}}{g} *(\gamma \#(1-\text { group }))}{\kappa_{f}^{-1}+\gamma_{f g 2}}\right)-\frac{\left(\gamma_{f g 2}-\gamma_{f g 1}\right)}{\left(\kappa_{f}^{-1}+\gamma_{f g 2}\right)^{2}} *\left(\frac{X^{\prime}}{I_{f}-g} *(\gamma \# \text { group })\right)
\end{aligned}
$$

and 


$$
\begin{aligned}
& \frac{\partial Y}{\partial \beta}= \\
& {\left[\left(\frac{\frac{X^{\prime}}{I_{f}-g} *(X \#(\gamma \# \text { group }))-\frac{X^{\prime}}{g} *(X \#(\gamma \#(1-\text { group })))}{\kappa_{f}^{-1}+\gamma_{f g 2}}\right)-\right.} \\
& \left(\frac{1}{\kappa_{f}^{-1}+\gamma_{f g 2}}\right)^{2}\left(\frac{X^{\prime}}{I_{f}-g} *(\gamma \# \text { group })-\frac{X^{\prime}}{g} *(\gamma \#(1-\text { group }))\right) *\left(\frac{X^{\prime}}{g} *(\gamma \# \text { group })\right)^{\prime}- \\
& \left(\frac{1}{\kappa_{f}^{-1}+\gamma_{f g 2}}\right)^{2}\left(\frac{X^{\prime}}{I_{f}-g} *(\gamma \# \text { group })\right) *\left(\frac{X^{\prime}}{I_{f}-g} *(\gamma \# \text { group })-\frac{X^{\prime}}{g} *(\gamma \#(1-\text { group }))\right)^{\prime}+ \\
& \left(\frac{2\left(\gamma_{f g 2}-\gamma_{f g 1}\right)}{\left(\kappa_{f}^{-1}+\gamma_{f g 2}\right)^{3}}\right) *\left(\frac{X^{\prime}}{I_{f}-g} *(\gamma \# \text { group })\right) *\left(\frac{X^{\prime}}{I_{f}-g} *(\gamma \# \text { group })\right)^{\prime}- \\
& \left.\frac{\left(\gamma_{f g 2}-\gamma_{f g 1}\right)}{\left(\kappa_{f}^{-1}+\gamma_{f g 2}\right)^{2}} *\left(\frac{X^{\prime}}{I_{f}-g} *(X \#(\gamma \# \text { group }))\right)\right] \text {. }
\end{aligned}
$$

